\title{
UN PROBLEMA BIEN PUESTO EN EL SENTIDO DE HADAMARD PARA UNA ECUACIÓN DE VIGA NO LINEAL
}

\author{
José Luyo Sánchez ${ }^{1}$
}

\begin{abstract}
RESUMEN.- Consideramos un sistema dinámico que modela las vibraciones no lineales de una viga prismática y uniforme de longitud finita y que considera los términos de aceleración longitudinal. Demostraremos que el sistema es bien puesto en el sentido de Hadamard para soluciones fuertes.
\end{abstract}

PALABRAS CLAVE.- Ecuación de viga no lineal. Sistema unidimensional de von Kárman.

\section{A PROBLEM WELLPOSED IN THE SENSE OF HADAMARD FOR AN NONLINEAR BEAM EQUATION}

\begin{abstract}
We consider a dynamical system which is a model for nonlinear vibrations of an uniform prismatic beam of length L, taking into account the inline accelerations terms. We show that the problem is wellposed in the sense of Hadamard for strong solutions.
\end{abstract}

KEY WORDS.- Nonlinear beam equations. One-dimensional von Kárman system.

\section{Introducción}

En este trabajo estudiamos un modelo dinámico que describe los efectos de estiramientos horizontales y oscilaciones verticales de una viga uniforme y prismática de longitud $L$, cuyos extremos se encuentran presos. Este está descrito por el sistema

$$
\left\{\begin{array}{c}
\rho A u_{t t}-E A\left[u_{x}+\frac{1}{2}\left(w_{x}\right)^{2}\right]_{x}=0, \text { en }(0, L) \times(0, \infty), \\
\rho A w_{t t}-\rho I w_{x x t t}+E I w_{x x x x}-E A\left[w_{x}\left(u_{x}+\frac{1}{2}\left(w_{x}\right)^{2}\right)\right]_{x}=0, \text { en }(0, L) \times(0, \infty), \\
u(0, t)=u(L, t)=0, w(0, t)=w(L, t)=w_{x}(0, t)=w_{x}(L, t)=0, \forall t \geq 0 .
\end{array}\right.
$$

En estas ecuaciones, las variables $u=u(x, t), w=w(x, t)$ representan respectivamente los desplazamientos horizontales y verticales de la viga. El parámetro constante $\mathrm{A}$, representa el área de la sección transversal de la viga, $E$ es el módulo de Young del material, $I$ el momento de inercia y el producto $E I$ es el módulo de rigidez de flexibilidad. Se hace là suposición de que las secciones transversales planas permanecen siempre planas y perpendiculares al eje longitudinal de la viga después de la deformación.

\footnotetext{
${ }^{1}$ Universidad Nacional Mayor de San Marcos. Facultad de Ciencias Matemáticas. e-mail: luyo@lncc.br
} 
J. Lagnese y G. Leugering en [9] nos presentan la formulación de este modelo de viga (1.1), conocido también en la literatura como el análogo unidimensional del sistema dinámico de Von Kárman. En este artículo, resultados de existencia, unicidad y estabilización son mostrados, haciendo uso de la teoría de semigrupos, para ciertas condiciones de frontera; sin embargo esto demandó el uso de operadores diferenciales inversos, herramienta que en este trabajo evitamos.

J. Puel y M. Tucsnak [16] considerando el sistema dinámico completo de Von Kárman, nos presentan resultados de existencia global de soluciones fuertes para un modelo no lineal de una placa. El método usado se basó principalmente en métodos de punto fijo en conjunción con limitaciones $a$ priori.

Es así que a partir de estos trabajos, tomamos como objeto de estudio el modelo dinámico no lineal de viga (1) presentado en [9] y adaptamos el método usado en [16] para demostrar que el modelo es bien puesto en el sentido de Hadamard para soluciones fuertes, es decir, que el sistema tiene solución, esta es única y depende continuamente de sus datos iniciales.

Para obtener estos resultados linealizamos el sistema, obteniendo una ecuación de onda y una ecuación de viga, las cuales resolvemos usando resultados clásicos de la teoría de semigrupos, posteriormente con el método de punto fijo recuperamos el sistema y a su vez encontramos una solución local del problema; resultados clásicos de continuidad nos garantizarán la existencia global de la solución. Finalmente usando el método de la energía demostramos que la solución es única y esta depende continuamente de los datos iniciales. La teoría usada en este trabajo son resultados usuales del análisis funcional, la teoría de semigrupos, que encontramos en el texto de A. Pazy [12], interpolación de operadores y espacios de Sobolev, que hallamos en el texto de J. Lions y E. Magenes [11]. La notación es la usual, como la que aparece en estos textos.

\section{Preliminares y Notaciones}

Sean $u=u(x, t)$ y $w=w(x, t)$, con $(x, t) \in[0, L] \times[0, \infty)$, variables reales que representan los desplazamientos horizontales y verticales de la viga respectivamente.

Para simplificar notaciones normalizamos los parámetros propios del material en la ecuación (1.1), iguales a 1.

Consideremos el sistema

$$
(P)\left\{\begin{array}{c}
u_{t t}-\left[u_{x}+\frac{1}{2}\left(w_{x}\right)^{2}\right]_{x}=0, \text { en }(0, L) \times(0, \infty), \\
w_{t t}-w_{x x t t}+w_{x x x x}-\left[w_{x}\left(u_{x}+\frac{1}{2}\left(w_{x}\right)^{2}\right]_{x}=0, \text { en }(0, L) \times(0, \infty),\right. \\
u(0, t)=u(L, t)=0, w(0, t)=w(L, t)=w_{x}(0, t)=w_{x}(L, t)=0, \forall t \geq 0 \\
u(x, 0)=u_{0}(x), u_{t}(x, 0)=u_{1}(x), w(x, 0)=w_{0}(x), w_{t}(x, 0)=w_{1}(x), \text { en }(0, L) .
\end{array}\right.
$$

La energía total asociada al sistema $(P)$ está dada por

$$
E(t)=\frac{1}{2} \int_{0}^{L}\left\{\left|u_{t}\right|^{2}+\left|w_{t}\right|^{2}+\left|w_{x t}\right|^{2}+\left|w_{x x}\right|^{2}\right\} d x+\frac{1}{2} \int_{0}^{L}\left(u_{x}+\frac{1}{2}\left(w_{x}\right)^{2}\right)^{2} d x
$$

Definición. Decimos que el sistema (P) tiene una solución global fuerte $\{u, w\}$, si

$$
\begin{aligned}
& u \in C\left(0, \infty ; H^{2}(0, L) \cap H_{0}^{1}(0, L)\right) \cap C^{1}\left(0, \infty ; H_{0}^{1}(0, L)\right) \cap C^{2}\left(0, \infty ; L^{2}(0, L)\right), \\
& w \in C\left(0, \infty ; H^{3}(0, L) \cap H_{0}^{2}(0, L)\right) \cap C^{1}\left(0, \infty ; H_{0}^{2}(0, L)\right) \cap C^{2}\left(0, \infty ; H_{0}^{1}(0, L)\right),
\end{aligned}
$$


y satisface la ecuación $(P)$ en el siguiente sentido:

$$
\left\{\begin{array}{c}
\left(u_{t t}, \varphi\right)+\left(u_{x}, \varphi_{x}\right)+\frac{1}{2}\left(\left(w_{x}\right)^{2}, \varphi_{x}\right)=0, \forall \varphi \in H_{0}^{1}(0, L), \\
\left(w_{t t}, \psi\right)+\left(w_{x t t}, \psi_{x}\right)+\left(w_{x x}, \psi_{x x}\right)+\left(w_{x}\left(u_{x}+\frac{1}{2}\left(w_{x}\right)^{2}\right), \psi_{x}\right)=0, \forall \psi \in H_{0}^{2}(0, L), \\
u(x, 0)=u_{0}(x), u_{t}(x, 0)=u_{1}(x), w(x, 0)=w_{0}(x), w_{t}(x, 0)=w_{1}(x), \text { en }(0, L) .
\end{array}\right.
$$

En estas dos primeras ecuaciones ( , ) representa el producto interno en $L^{2}(0, L)$.

La energía total de la viga está dada por

$$
E(t)=\frac{1}{2} \int_{0}^{L}\left\{\left|u_{t}\right|^{2}+\left|w_{t}\right|^{2}+\left|w_{x t}\right|^{2}+\left|w_{x x}\right|^{2}\right\} d x+\frac{1}{2} \int_{0}^{L}\left(u_{x}+\frac{1}{2}\left(w_{x}\right)^{2}\right)^{2} d x
$$

Lema 2.1. Sea $\{u, w\}$ una solución fuerte de $(P)$ entonces se tiene que

$$
E(t)=E(0), \forall t \geq 0
$$

A continuación enunciamos un resultado de la teoría de semigrupos, cuya demostración podemos hallar en [5] o [12] ésta concentra resultados de existencia de soluciones de ecuaciones así como estimativas de sus soluciones, las cuales haremos uso en el transcurso del trabajo.

Teorema 2.1 Sea $X$ un espacio de Banach y $A$ un operador maximal disipativo definido en $X$ que es generador infinitesimal de un semigrupo de contracciones $\{T(t)\}$ en $X$. si $f \in C^{1}([0, T], X), u_{0} \in D(A)$, entonces el problema de valor inicial

$$
\frac{d}{d t} u=A u+f(t), \quad u(0)=u_{0},
$$

admite una única solución u tal que,

$$
u \in C^{1}([0, T], X) \cap C([0, T], D(A)) .
$$

La solución puede ser expresada en la forma,

$$
\begin{gathered}
u(t)=T(t) u_{0}+\int_{0}^{t} T(t-s) f(s) d s, \\
\frac{d}{d t} u(t)=T(t) A u_{0}+T(t) f(0)+\int_{0}^{t} T(t-s) f_{t}(s) d s,
\end{gathered}
$$

además u y ut satisfacen,

$$
\begin{gathered}
\|u(t)\|_{X} \leq\left\|u_{0}\right\|_{X}+\int_{0}^{t}\|f(s)\|_{X} d s, \\
\left\|u_{t}(t)\right\|_{X} \leq\left\|A u_{0}\right\|_{X}+\|f(0)\|_{X}+\int_{0}^{t}\left\|f_{t}(s)\right\|_{X} d s .
\end{gathered}
$$




\section{El problema linealizado}

Linealizamos el sistema (P) con el objetivo de desacoplarlo y luego encontrar solución de los problemas linealizados. Para esto sustituimos en el sistema la expresión $\frac{1}{2}\left(w_{x}\right)^{2}$ por una función $f=f(x, t)$ y $w_{x}\left(u_{x}+\frac{1}{2}\left(w_{x}\right)^{2}\right)$ por una función $g=g(x, t)$, luego tenemos

$$
\left\{\begin{array}{c}
u_{t t}-u_{x x}=f_{x} \\
w_{t t}-w_{x x t t}+w_{x x x x}=g_{x}
\end{array}\right.
$$

Formulamos el siguiente problema

$$
(P 1)\left\{\begin{array}{c}
u_{t t}-u_{x x}=f_{x}, \text { en }(0, L) \times(0, \infty), \\
u(0, t)=u(L, t)=0, t \geq 0, f=f(x, t):(0, L) \times(0, T) \rightarrow \mathbb{R}, \\
u(x, 0)=u_{0}, u_{t}(x, 0)=u_{1}, \text { en }(0, L) .
\end{array}\right.
$$

Este problema (P1) es una ecuación de onda no homogénea con condiciones de Dirichlet, resolvemos este problema usando teoría de semigrupos.

Teorema 3.1. Dados $u_{0} \in H^{2} \cap H_{0}^{1}, u_{1} \in H_{0}^{1}, y f \in C^{1}\left([0, T], H_{0}^{1}(0, L)\right)$, entonces el problema (P1) admite una única solución u tal que

$$
u \in C^{2}\left([0, T], H^{2}(0, L) \cap H_{0}^{1}(0, L)\right) \cap C^{1}\left([0, T], H_{0}^{1}(0, L) \cap C\left([0, T], L^{2}(0, L)\right)\right.
$$

satisface las condiciones iniciales del problema y existe una constante $C>0$, independiente de $u$ y $f$ tal que

$$
\begin{aligned}
\|u(t)\|_{H^{2} \cap H_{0}^{1}} & +\left\|u_{t}(t)\right\|_{H_{0}^{1}}+\left\|u_{t t}(t)\right\|_{L^{2}} \leq\left\|u_{1}\right\|_{H_{0}^{1}}+\left\|u_{0}\right\|_{H^{2} \cap H_{0}^{1}}+\|f(0)\|_{H_{0}^{1}}+ \\
& +\int_{0}^{t}\left\|f_{t}(s)\right\|_{H_{0}^{1}} d s, \forall t \in[0, T]
\end{aligned}
$$

Demostración. De (P1) tenemos el problema equivalente

$$
\left(\begin{array}{c}
u \\
u_{t}
\end{array}\right)_{t}=\left(\begin{array}{c}
u_{t} \\
u_{x x}
\end{array}\right)+\left(\begin{array}{c}
0 \\
f_{x}
\end{array}\right)
$$

Sea $v=u_{t}$, entonces

$$
\left(\begin{array}{l}
u \\
v
\end{array}\right)_{t}=A\left(\begin{array}{l}
u \\
v
\end{array}\right)+\left(\begin{array}{c}
0 \\
f_{x}
\end{array}\right)
$$

Donde $A$ es un operador definido en el espacio $H_{0}^{1} \times L^{2}$ con dominio $D(A)=H_{0}^{2} \cap H_{0}^{1}$. 


$$
A: D(A) \rightarrow H_{0}^{1} \times L^{2}
$$

y está definido por

$$
A\left(\begin{array}{l}
u \\
v
\end{array}\right)=\left(\begin{array}{c}
v \\
u_{x x}
\end{array}\right), \forall u \in H^{2} \cap H_{0}^{1}, v \in H_{0}^{1}
$$

En el espacio $H_{0}^{1} \times L^{2}$ definimos el producto interno

$$
\left(\left(\begin{array}{l}
u \\
v
\end{array}\right),\left(\begin{array}{c}
\tilde{u} \\
\tilde{v}
\end{array}\right)\right)_{H_{0}^{1} \times L^{2}}=\left(u_{x}, \tilde{u}_{x}\right)+(v, \tilde{v}), \forall(u, v),(\tilde{u}, \tilde{v}) \in H_{0}^{1} \times L^{2}
$$

Afirmamos que el operador $A$, definido arriba, es generador infinitesimal de un semigrupo de contracciones en $H_{0}^{1} \times L^{2}$.

En efecto; será suficiente demostrar que el operador $A$ es maximal disipativo, ver [12]. Dado $\left(\begin{array}{l}u \\ v\end{array}\right) \in D(A)$, entonces de la integración por partes se sigue que

$$
\left(A\left(\begin{array}{l}
u \\
v
\end{array}\right),\left(\begin{array}{l}
u \\
v
\end{array}\right)\right)_{H_{0}^{1} \times L^{2}}=\left(v_{x}, u_{x}\right)+\left(u_{x x}, v\right)=0 .
$$

Por otro lado, dado $\left(\begin{array}{l}f \\ g\end{array}\right) \in H_{0}^{1} \times L^{2}$, debemos encontrar $\left(\begin{array}{l}u \\ v\end{array}\right) \in D(A)$ tal que

$$
(I-A)\left(\begin{array}{l}
u \\
v
\end{array}\right)=\left(\begin{array}{l}
f \\
g
\end{array}\right)
$$

es decir

$$
\left\{\begin{array}{r}
u-v=f \\
v-u_{x x}=g
\end{array}\right.
$$

luego bastará resolver el problema elíptico

$$
\text { (E) }\left\{\begin{array}{c}
2 u-u_{x x}=f+g \in L^{2}(0, L) \\
u(0, t)=u(L, t)=0
\end{array}\right.
$$

En efecto, sea la forma bilineal $a: H_{0}^{1} \times H_{0}^{1} \rightarrow L^{2}$, definida por

$$
a(u, v)=\left(u_{x}, v_{x}\right)+2(u, v), \forall u, v \in H_{0}^{1}
$$

El problema variacional asociado al problema (E) consiste en encontrar solución para 


$$
a(u, \psi)=(f+g, \psi), \forall \psi \in H_{0}^{1} .
$$

La forma bilineal a es continua y coerciva. En efecto:

Continua: Dados $u, v \in H_{0}^{1}$, de la desigualdad de Cauchy - Schwarz

$$
|a(u, v)| \leq\left\|u_{x}\right\|_{L^{2}}\left\|v_{x}\right\|_{L^{2}}+2\|u\|_{L^{2}}\|v\|_{L^{2}} .
$$

entonces por la desigualdad de Poincaré tenemos

$$
|a(u, v)| \leq C\left\|u_{x}\right\|_{L^{2}}\left\|v_{x}\right\|_{L^{2}}=C\|u\|_{H_{0}^{1}}\|v\|_{H_{0}^{1}} \forall u, v \in H_{0}^{1}
$$

Coerciva: Deja $u \in H_{0}^{1}$, entonces

$$
a(u, u)=\left\|u_{x}\right\|_{L^{2}}^{2}+2\|u\|_{L^{2}}^{2} \geq\|u\|_{H_{0}^{1}}^{2} .
$$

Por lo tanto, por el Teorema de Lax-Milgram [7], existe una única solución $u$ del problema variacional (3.6) asociado al problema (E) tal que $u \in H_{0}^{1}$ y por la regularidad elíptica se sigue que $u \in H^{2}$, luego $u \in H^{2} \cap H_{0}^{1}$. Como $v=u-f$ entonces $v \in H_{0}^{1}$. Por lo tanto, de la teoría de semigrupos, se tiene que el operador $A$ es generador infinitesimal de un semigrupo de contracciones $\{T(t)\}$.

Por otro lado, del hecho que $f \in C^{1}\left([0, T], H_{0}^{1}\right)$ entonces

$$
\left(\begin{array}{c}
0 \\
f_{x}
\end{array}\right) \in C^{1}\left([0, T], H_{0}^{1} \times L^{2}\right) .
$$

Luego de los resultados establecidos, el problema (3.5) tiene una única solución

$$
\left(\begin{array}{l}
u \\
v
\end{array}\right) \in C^{1}\left([0, T], H_{0}^{1} \times L^{2}\right) \times C\left([0, T], H^{2} \cap H_{0}^{1} \times H_{0}^{1}\right),
$$

es decir

$$
u \in C\left([0, T], H^{2} \cap H_{0}^{1}\right) \cap C^{1}\left([0, T], H_{0}^{1}\right) \cap C^{2}\left([0, T], L^{2}\right) .
$$

Por otro lado aplicamos la desigualdad (2.3) y tenemos

$$
\left\|u_{t}(t)\right\|_{H_{0}^{1}}+\left\|u_{t t}(t)\right\|_{L^{2}} \leq\left\|u_{1}\right\|_{H_{0}^{1}}+\left\|u_{0}\right\|_{H^{2} \cap H_{0}^{1}}+\|f(0)\|_{H_{0}^{1}}+\int_{0}^{t}\left\|f_{t}(s)\right\|_{H_{0}^{1}} d s
$$

Del problema (P1), 


$$
-u_{x x}=-u_{t t}+f_{x} \in L^{2}
$$

de donde obtenemos que

$$
\|u\|_{H^{2} \cap H_{0}^{1}} \leq\left\|u_{t t}\right\|_{L^{2}}+\|f\|_{H_{0}^{1}} .
$$

Observamos que $f(t)=f(0)+\int_{0}^{t} f_{t}(s) d s$ en $H_{0}^{1}$, luego deducimos que

$$
\|f(t)\|_{H_{0}^{1}}=\|f(0)\|_{H_{0}^{1}}+\int_{0}^{t}\left\|f_{t}(s)\right\|_{H_{0}^{1}} d s
$$

usando en (3.10) este último resultado y (3.9) tenemos

$$
\|u\|_{H^{2} \cap H_{0}^{1}} \leq\left\|u_{1}\right\|_{H_{0}^{1}}+\left\|u_{0}\right\|_{H^{2} \cap H_{0}^{1}}+\|f(0)\|_{H_{0}^{1}}+\int_{0}^{t}\left\|f_{t}(s)\right\|_{H_{0}^{1}} d s .
$$

Por lo tanto, asumando (3.9) y (3.11) se sigue (3.4).

Consideremos ahora el segundo problema

$$
\left\{\begin{array}{c}
w_{t t}-w_{x x t t}+w_{x x x x}=g_{x}, \text { en }(0, L) \times(0, T) \\
w(0, t)=w(L, t)=w_{x}(0, t)=w_{x}(L, t)=0, \forall t \geq 0, \\
w(x, 0)=w_{0}(x), w_{t}(x, 0)=w_{1}(x), \text { en }(0, L), \\
g=g(x, t):(0, L) \times(0, T) \rightarrow \mathbb{R} .
\end{array}\right.
$$

Cuya forma variacional definimos como

$$
(P 2)\left\{\begin{array}{c}
\left(w_{t t}, \psi\right)+\left(w_{x t t}, \psi_{x}\right)+\left(w_{x x}, \psi_{x x}\right)+\left(g, \psi_{x}\right)=0, \forall \psi \in H_{0}^{2}, \\
w(0, t)=w(L, t)=w_{x}(0, t)=w_{x}(0, L)=0, \quad \forall t \geq 0, \\
w(x, 0)=w_{0}(x), w_{t}(x, 0)=w_{1}(x), \text { en }(0, L) .
\end{array}\right.
$$

Establecemos ahora, condiciones para resolver (P2).

Definición. Definimos el conjunto

$$
D(A)=\left\{w \in H_{0}^{2} / \exists y \in H_{0}^{1} \text { talque }\left(w_{x x}, \psi_{x x}\right)=\left(y_{x}, \psi_{x}\right)+(y, \psi), \forall \psi \in H_{0}^{2}\right\}
$$

Lema 3.1 En estas condiciones se tiene que $D(A)=H^{3} \cap H_{0}^{2}$.

Demostración. Sea $w \in H^{3} \cap H_{0}^{2}$, luego $w_{x x x} \in L^{2}$. Consideremos el problema variacional

$$
\left(-w_{x x x}, \psi_{x}\right)=\left(y_{x}, \psi_{x}\right)+(y, \psi), \forall \psi \in H_{0}^{1} .
$$


Entonces por el Teorema de Lax-Milgram existe una única $y \in H_{0}^{1}$ tal que satisface (3.12). En particular tenemos que

$$
\left(-w_{x x}, \psi_{x}\right)=\left(y_{x}, \psi_{x}\right)+(y, \psi), \forall \psi \in H_{0}^{2}
$$

integrando por partes se sigue que

$$
\left(w_{x x}, \psi_{x x}\right)=\left(y_{x}, \psi_{x}\right)+(y, \psi), \forall \psi \in H_{0}^{2}
$$

luego $w \in D(A)$. Lo que demuestra también que $D(A)$ no es vacío.

Sea ahora $w \in D(A)$, entonces por definición existe $y \in H_{0}^{1}$ tal que

$$
\left(w_{x x}, \psi_{x x}\right)=\left(y_{x}, \psi_{x}\right)+(y, \psi), \forall \psi \in H_{0}^{2} .
$$

Sea la aplicación $F: H_{0}^{1} \rightarrow R$ definida por

$$
\langle F, \psi\rangle=\left(y_{x}, \psi_{x}\right)+(y, \psi), \forall \psi \in H_{0}^{1}
$$

entonces $F$ es lineal y continua en $H_{0}^{1}$, luego $F \in H^{-1}$. Por tanto de (3.13) tenemos que

$$
\left(w_{x x}, \psi_{x x}\right)=(F, \psi), \forall \psi \in H_{0}^{2} .
$$

A continuación usaremos la teoría de interpolación para resolver este problema variacional.

Consideremos las siguientes alternativas:

I ) $\mathrm{Si} F \in L^{2}$, entonces tenemos

$$
\left(w_{x x}, \psi_{x x}\right)=(F, \psi), \forall \psi \in H_{0}^{2},
$$

luego por el Teorema de Lax-Milgram se tiene que $w \in H_{0}^{2}$, por regularidad elíptica [4], tenemos $w \in H^{4}$, por lo tanto $w \in H^{4} \cap H_{0}^{2}$ y $w_{x x x x x}=F$, de donde se obtiene que

$$
\|w\|_{H^{4} \cap H_{0}^{2}} \leq\|F\|
$$

Asociamos al problema (3.16) el siguiente operador lineal

$$
\begin{aligned}
T: L^{2} & \rightarrow H^{4} \cap H_{0}^{2} \\
F & \rightarrow w
\end{aligned}
$$

tal que a cada $F \in L^{2}$ asocia $w \in H^{4} \cap H_{0}^{2}$ solución del problema (3.16). Luego de (3.17) tenemos que 


$$
\|T(F)\|_{H^{4} \cap H_{0}^{2}} \leq\|F\|_{L^{2}}
$$

y además se tiene que

$$
\|T\|_{\mathcal{L}\left(L^{2}, H^{-2}\right)} \leq 1
$$

II ) $\mathrm{Si} F \in H^{-2}$, entonces tenemos

$$
\left(w_{x x}, \psi_{x x}\right)=\langle F, \psi\rangle, \forall \psi \in H_{0}^{2},
$$

por el Teorema de Lax-Milgram se sigue que $w \in H_{0}^{2}$. Luego de (3.18) tenemos

$$
\|w\|_{H_{0}^{2}} \leq C\|F\|_{H^{-2}}
$$

donde $C>0$ es una constante independiente de $w$ y $F$. Del mismo modo asociamos al problema (3.18) el siguiente operador lineal

$$
\begin{aligned}
T: H^{-2} & \rightarrow H_{0}^{2} \\
F & \rightarrow w
\end{aligned}
$$

tal que a cada $F \in H^{-2}$ asocia $w \in H_{0}^{2}$ solución del problema (3.18). Luego de (3.19) tenemos que

$$
\|T(F)\|_{H^{4} \cap H_{0}^{2}} \leq C\|F\|_{L^{2}}
$$

y además se tiene que

$$
\|T\|_{\mathcal{L}\left(H^{4} \cap H_{0}^{2}, H_{0}^{2}\right)} \leq C .
$$

De (I) y (II) tenemos que el operador $T$ es tal que

$$
T \in \mathfrak{L}\left(L^{2}, H^{4} \cap H_{0}^{2}\right) \cap \mathfrak{L}\left(H^{-2}, H_{0}^{2}\right) .
$$

De la teoría de interpolación de espacios de Sobolev [11], se tiene que

$$
\left[L^{2}, H^{-2}\right]_{\frac{1}{2}}=H^{-1} y\left[H^{4} \cap H_{0}^{2}, H_{0}^{2}\right]_{\frac{1}{2}}=H^{3} \cap H_{0}^{2},
$$

luego el operador $T$ es tal que

$$
T \in \mathfrak{L}\left(H^{-1}, H^{3} \cap H_{0}^{2}\right),
$$

y por [7] este satisface

$$
\|T\|_{\mathcal{L}\left(H^{-1}, H^{3} \cap H_{0}^{2}\right)} \leq\|T\|_{\mathfrak{L}\left(L^{2}, H^{-2}\right)}^{\frac{1}{2}}\|T\|_{\mathcal{L}\left(H^{4}, H_{0}^{2}, H_{0}^{2}\right)}^{\frac{1}{2}} .
$$

Por tanto, como en el problema inicial (3.15) tenemos que $F \in H^{-1}$, entonces $w=T(F) \in H^{3} \cap H_{0}^{2}$ es solución del problema (3.15) y es tal que $\|w\|_{H^{3} \cap H_{0}^{2}} \leq C\left\|_{F}\right\|_{H^{-1}}$. Como $\mathrm{F}$ está definido por la 
ecuación (3.14), entonces existe una constante $C>0$ independiente de $F$ y $f$ tal que

$$
\|F\|_{H^{-1}}=\sup _{\eta \neq 0} \frac{\left|\langle F, \eta\rangle_{H^{-1}, H_{0}^{1}}\right|}{\|\eta\|_{H_{0}^{1}}} \leq \frac{\|y\|\|\eta\|+\left\|y_{x}\right\|\left\|\eta_{x}\right\|}{\|\eta\|_{H_{0}^{1}}} \leq C\|y\|_{H_{0}^{1}} .
$$

Luego concluimos que $\|w\|_{H^{3} \cap H_{0}^{2}} \leq C\|y\|_{H_{0}^{1}}$.

Sea el operador $\mathbb{A}$ definido en el espacio $H_{0}^{2} \cap H_{0}^{1}$, con dominio

$$
D(\mathbb{A})=\left\{\left(\begin{array}{c}
w \\
v
\end{array}\right) \in D(A) \times H_{0}^{2}\right\}
$$

tal que

$$
\mathbb{A}\left(\begin{array}{l}
w \\
v
\end{array}\right)=\left(\begin{array}{c}
v \\
-A w
\end{array}\right), \forall(w, v) \in D(\mathbb{A})
$$

En el espacio $H_{0}^{2} \times H_{0}^{1}$ definimos el siguiente producto interno

$$
\left(\left(\begin{array}{c}
w \\
v
\end{array}\right),\left(\begin{array}{c}
\tilde{w} \\
\tilde{v}
\end{array}\right)\right)_{H_{0}^{2} \times H_{0}^{1}}=\left(w_{x x}, \tilde{w}_{x x}\right)+\left(v_{x}, \tilde{v}_{x}\right)+(v, \tilde{v}), \forall(w, v),(\tilde{w}, \tilde{v}) \in H_{0}^{2} \times H_{0}^{1}
$$

Lema 3.2 El operador $\mathbb{A}: D(\mathbb{A}) \rightarrow H_{0}^{2} \times H_{0}^{1}$, definido anteriormente, es el generador infinitesimal de un semigrupo de contracciones en $H_{0}^{2} \times H_{0}^{1}$.

Demostración. Será suficiente demostrar que $\mathbb{A}$ es maximal disipativo [7], para lo cual calcularemos el producto interno

$$
\begin{array}{r}
\left(\mathbb{A}\left(\begin{array}{c}
w \\
v
\end{array}\right),\left(\begin{array}{c}
w \\
v
\end{array}\right)\right)_{H_{0}^{2} \times H_{0}^{1}}=\left(\left(\begin{array}{c}
v \\
-A w
\end{array}\right),\left(\begin{array}{c}
w \\
v
\end{array}\right)\right)_{H_{0}^{2} \times H_{0}^{1}}= \\
=\left(v_{x x}, w_{x x}\right)+\left((-A w)_{x}, v_{x}\right)+((-A w), v)=0
\end{array}
$$

Por lo tanto

$$
\left(\mathbb{A}\left(\begin{array}{l}
w \\
v
\end{array}\right),\left(\begin{array}{c}
w \\
v
\end{array}\right)\right)_{H_{0}^{2} \times H_{0}^{1}}=0, \quad \forall\left(\begin{array}{l}
w \\
v
\end{array}\right) \in D(\mathbb{A})
$$

Luego $\mathbb{A}$ es disipativo en $H_{0}^{2} \times H_{0}^{1}$. Dado $(f, g) \in H_{0}^{2} \times H_{0}^{1}$, debemos encontrar $(w, v) \in D(\mathbb{A})$ tal que 


$$
\begin{gathered}
\left(\mathbb{A}\left(\begin{array}{l}
w \\
v
\end{array}\right),\left(\begin{array}{l}
f \\
g
\end{array}\right)\right) \text { es decir }\left(\left(\begin{array}{c}
v \\
-A w
\end{array}\right),\left(\begin{array}{c}
f \\
g
\end{array}\right)\right), \\
v=f \in H_{0}^{2} \text { entonces } v \in H_{0}^{2} \quad \text { y }-A w=g .
\end{gathered}
$$

Sea $y=-g \in H_{0}^{1}$, tenemos que demostrar que existe $w \in D(A)$ tal que

$$
\left(w_{x x}, \psi_{x x}\right)=(y, \psi)+\left(y_{x}, \psi_{x}\right), \forall \psi \in H_{0}^{2}
$$

Sea $y^{*} \in H^{-1}$, definido como $\left\langle y^{*}, \psi\right\rangle=(y, \psi)+\gamma(\nabla y, \nabla \psi), \forall \psi \in H_{0}^{1}$.

Entonces tenemos el siguiente problema variacional

$$
\left(w_{x x}, \psi_{x x}\right)=\left(y^{*}, \psi\right), y^{*} \in H^{-1}, \forall \psi \in H_{0}^{2},
$$

usando la teoría de interpolación mostramos que el problema admite una única solución $w \in D(\mathbb{A})$.

De esta forma, existe $\left(\begin{array}{l}w \\ v\end{array}\right) \in D(\mathbb{A})$ tal que

$$
\mathbb{A}\left(\begin{array}{l}
w \\
v
\end{array}\right)=\left(\begin{array}{l}
f \\
g
\end{array}\right) \in H_{0}^{2} \times H_{0}^{1}
$$

Por lo tanto $\mathbb{A}$ es un operador maximal disipativo.

Teorema 3.2 Dados $w_{0} \in H^{3} \cap H_{0}^{2}, w_{1} \in H_{0}^{2}, g \in C^{1}\left([0, T], L^{2}\right)$, entonces el problema (P2) admite una única solución $w$ tal que

$$
w \in C\left([0, T], H^{3} \cap H_{0}^{2}\right) \cap C^{1}\left([0, T], H_{0}^{2}\right) \cap C\left([0, T], H_{0}^{1}\right),
$$

satisfaciendo las condiciones iniciales del problema, además existe una constante $C>0$ independiente de w y g tal que

$$
\|w\|_{H^{3} \cap H_{0}^{2}}+\left\|w_{t}\right\|_{H_{0}^{2}}+\left\|w_{t t}\right\|_{H_{0}^{1}} \leq\left\|w_{1}\right\|_{H_{0}^{2}}+\left\|A w_{0}\right\|_{H_{0}^{1}}+\|g(0)\|_{L^{2}}+\int_{0}^{t}\left\|g_{t}(s)\right\|_{L^{2}} d s .
$$

Demostración. Usaremos resultados de la teoría de Semigrupos para demostrar este resultado. Definamos la función $\tilde{g}=\tilde{g}(x, t)$ como la solución del siguiente problema variacional

$$
(\tilde{g}, \psi)+\left(\tilde{g}_{x}, \psi_{x}\right)=\left(g, \psi_{x}\right), \forall \psi \in H_{0}^{1}
$$

Entonces, usando el Teorema de Lax-Milgram mostramos que $\tilde{g} \in C^{1}\left([0, T] ; H_{0}^{1}\right)$. Además deducimos de (3.20) las siguientes desigualdades

$$
\|\tilde{g}(t)\|_{H_{0}^{1}} \leq C\|g(t)\|_{L^{2}} \text { y }\left\|\tilde{g}_{t}(t)\right\|_{H_{0}^{1}} \leq C\left\|g_{t}(t)\right\|_{L^{2}}, \forall t \in[0, T], C=\text { cte. }
$$


Sea $v=w_{t}$, definimos $Y=\left(\begin{array}{c}w \\ v\end{array}\right), Y_{0}=\left(\begin{array}{l}w_{0} \\ w_{1}\end{array}\right)$ y $G=\left(\begin{array}{l}0 \\ \tilde{g}\end{array}\right)$, con estas notaciones establecemos el siguiente problema de valor inicial

$$
\left\{\begin{array}{l}
Y_{t}=\mathbb{A} Y+G \\
Y(0)=Y_{0}
\end{array}\right.
$$

donde $Y_{0} \in D(\mathbb{A}), G \in C^{1}\left([0, T] ; H_{0}^{2} \times H_{0}^{1}\right)$. Luego, como $\mathbb{A}$ es el generador infinitesimal de un semigrupo de contracciones, entonces existe una única solución

$$
Y \in C([0, T] ; D(\mathbb{A})) \times C^{1}\left([0, T] ; H_{0}^{2} \times H_{0}^{1}\right) .
$$

es decir, como $v=w_{t}$, entonces

$$
w \in C\left([0, T] ; H^{3} \cap H_{0}^{2}\right) \cap C^{1}\left([0, T] ; H_{0}^{2}\right) \cap C^{2}\left([0, T] ; H_{0}^{1}\right) .
$$

Por otro lado, usando el Teorema 2.1, tenemos

$$
\left\|w_{t}\right\|_{H_{0}^{2}}+\left\|w_{t t}\right\|_{H_{0}^{1}} \leq\left\|w_{1}\right\|_{H_{0}^{2}}+\left\|A w_{0}\right\|_{H_{0}^{1}}+\|\tilde{g}(0)\|_{H_{0}^{1}}+\int_{0}^{t}\left\|\tilde{g}_{t}(s)\right\|_{H_{0}^{1}} d s
$$

Usando (3.21) se tiene

$$
\left\|w_{t}\right\|_{H_{0}^{2}}+\left\|w_{t t}\right\|_{H_{0}^{1}} \leq\left\|w_{1}\right\|_{H_{0}^{2}}+\left\|A w_{0}\right\|_{H_{0}^{1}}+C\|g(0)\|_{L^{2}}+C \int_{0}^{t}\left\|g_{t}(s)\right\|_{L^{2}} d s .
$$

De la ecuación inicial (P2) tenemos

$$
\left(w_{x x}, \psi_{x x}\right)=\left(-w_{t t}, \psi\right)+\left(-w_{x t t}, \psi_{x}\right)+\left(g(t), \psi_{x}\right) .
$$

De la definición de $\tilde{g}$ en (3.20) tenemos que

$$
\left(w_{x x}, \psi_{x x}\right)=\left(-w_{t t}+\tilde{g}, \psi\right)+\left(-w_{x t t}+\tilde{g}_{x}, \psi_{x}\right)
$$

como $-w_{t t}(t)+\tilde{g}(t) \in H_{0}^{1}$, entonces usando la teoría de interpolación para espacios de Sobolev, tenemos

$$
\|w\|_{H^{3} \cap H_{0}^{2}} \leq\left\|w_{t t}\right\|_{H_{0}^{1}}+\|\tilde{g}\|_{H_{0}^{1}}
$$

Como $g(t)=g(0)+\int_{0}^{t} g(s)$ en $L^{2}$, entonces

$$
\|g(t)\|_{L^{2}} \leq\|g(0)\|_{L^{2}}+\int_{0}^{t}\|g(s)\|_{L^{2}} d s, \forall t \in[0, T] .
$$


Usando la desigualdad (3.21) en la expresión anterior, tenemos que

$\|\tilde{g}(t)\|_{H_{0}^{1}} \leq C\|g(0)\|_{L^{2}}+C \int_{0}^{t}\|g(s)\|_{L^{2}} d s, \forall t \in[0, T]$, donde $C$ es constante.

Ahora usando este último resultado y (3.22) en (3.23) tenemos que existe una constante $C>0$ tal que

$$
\|w\|_{H^{3} \cap H_{0}^{2}} \leq\left\|w_{1}\right\|_{H_{0}^{2}}+\left\|A w_{0}\right\|_{H_{0}^{1}}+C\|g(0)\|_{L^{2}}+C \int_{0}^{t}\left\|g_{t}(s)\right\|_{L^{2}} d s
$$

Finalmente, sumando en las desigualdades (3.22) y (3.24) concluimos con la demostración del Teorema.

\section{Análisis de los términos no lineales}

El análisis de los términos no lineales será básicamente resultado del hecho que en un dominio unidimensional $I \subset \mathbb{R}$ tenemos que $H^{m}(I) \mapsto L^{\infty}(I)$, para todo $m \geq 1$. Enunciamos los siguientes resultados:

Teorema 4.1 Si $m \geq 1$, entonces para todo $u, v \in H^{m}(I)$, el producto $u v$, definido puntualmente sobre I pertenece a $H^{m}(I)$ y existe una constante $C>0$ dependiendo apenas de $m$, tal que se satisface

$$
\|u v\|_{H^{m}} \leq C\|u\|_{H^{m}}\|v\|_{H^{m}}
$$

en particular $H^{m}(I)$ es un Álgebra de Banach conmutativa.

Demostración. Ver [1].

Lema 4.1 Dados $u \in H^{1}(I), v \in L^{2}(I)$ entonces $u v \in L^{2}$ existe una constante $C>0$ tal que $\|u v\|_{L^{2}} \leq C\|u\|_{H^{1}}\|v\|_{L^{2}}$

Lema 4.2 Dados $u \in H^{2}(I), v \in H^{1}(I)$ entonces $u v \in H^{1}(I)$ existe una constante $C>0$ tal que

$$
\|u v\|_{H^{1}} \leq C\left\|_{u}\right\|_{H^{2}}\|v\|_{H^{1}}
$$

Para abreviar términos, definamos los siguientes espacios

$$
\begin{gathered}
Y_{u}=C^{2}\left([0, T], H^{2}(0, L) \cap H_{0}^{1}(0, L)\right) \cap C^{1}\left([0, T], H_{0}^{1}(0, L)\right) \cap C\left([0, T], L^{2}(0, L)\right), \\
Y_{w}=C\left([0, T] ; H^{3} \cap H_{0}^{2}\right) \cap C^{1}\left([0, T] ; H_{0}^{2}\right) \cap C^{2}\left([0, T] ; H_{0}^{1}\right) .
\end{gathered}
$$

Observemos que $Y_{u}$ y $Y_{w}$ son los espacios solución de los problemas (P1) y (P2) respectivamente. 
Teorema 4.2 Sean $u \in Y_{u}, w \in Y_{w}$, definimos las funciones $f y$ g como

$$
f=\frac{1}{2}\left(w_{x}\right)^{2}, g=w_{x}\left(u_{x}+\frac{1}{2}\left(w_{x}\right)^{2}\right)
$$

Entonces

$$
\begin{gathered}
f \in C\left([0, T] ; H_{0}^{2}(0, L) \cap C^{1}\left([0, T], H_{0}^{1}(0, L)\right) \cap C^{2}\left([0, T] ; L^{2}(0, L)\right):=Y_{f},\right. \\
g \in C\left([0, T] ; H_{0}^{1}\right) \cap C^{1}\left([0, T] ; L^{2}(0, L)\right):=Y_{g}
\end{gathered}
$$

$y$ estas funciones satisfacen

$$
\begin{gathered}
\|f\|_{Y_{f}} \leq C\left\|_{w}\right\|_{Y_{u}}^{2}, \\
\|g\|_{Y_{g}} \leq C\left(\|u\|_{Y_{u}}\|w\|_{Y_{w}}+\|w\|_{Y_{w}}^{3}\right),
\end{gathered}
$$

donde $C>0$ es una constante que depende sólo de las constantes de inmersión.

Demostración. Consideramos los espacios $Y_{f}$ y $Y_{g}$ con sus normas usuales. Usando el Teorema 4.1 y del hecho que $w \in H_{0}^{2}$ deducimos que

$$
f=\frac{1}{2} w_{x} w_{x} \in H^{2} \cap H_{0}^{1}, \text { entonces } f \in C\left([0, T], H^{2} \cap H_{0}^{1}\right) .
$$

Tenemos también que

$$
\|f\|_{H^{2}} \leq C\left\|_{w}\right\|_{H^{3}}^{2} \text { y }\|f\|_{H^{1}} \leq C\left\|_{w}\right\|_{H_{0}^{2}}^{2}
$$

Como $f \in H_{0}^{1}$, entonces existe una constante $C>0$ tal que $\|f\|_{H_{0}^{1}} \leq C\|f\|_{H^{1}}$. Por lo tanto tenemos que existe una constante $C>0$ tal que

$$
\|f\|_{H^{2} \cap H_{0}^{1}} \leq C\|w\|_{Y_{w}}^{2}
$$

Ahora derivamos $f$ en relación a t, por el Lema 4.2 y como $w \in H_{0}^{2}$ tenemos

$$
f_{t}=w_{x} w_{t x} \in H_{0}^{1} \text {, entonces } f \in C^{1}\left([0, T], H_{0}^{1}\right)
$$

Luego

$$
\left\|f_{t}\right\|_{H^{1}} \leq\|w\|_{H^{2}}\left\|w_{t}\right\|_{H^{2}}
$$

Como $f_{t} \in H_{0}^{1}, w \in H_{0}^{2}$ y $w_{t} \in H_{0}^{2}$ entonces deducimos que existe una constante $C>0$ tal que

$$
\left\|f_{t}\right\|_{H_{0}^{1}} \leq C\left\|_{w}\right\|_{Y_{w}}\|w\|_{Y_{w}}
$$


Ahora usando el Teorema 4.1 y el Lema 4.2, se sigue que

$$
f_{t t}=w_{t x} w_{t x}+w_{x} w_{t t x} \in H^{1}+L^{2} \text {, entonces } f \in C^{2}\left([0, T], L^{2}\right)
$$

Luego usando el Lema 4.1 tenemos

$$
\left\|f_{t t}\right\|_{L^{2}} \leq C\left\|w_{t x} w_{t x}\right\|_{H^{1}}+C\left\|w_{x}\right\|_{H^{1}}\left\|w_{t t x}\right\|_{L^{2}}
$$

Luego como $w \in H_{0}^{2}$ y $w_{t t} \in H_{0}^{1}$, entonces se tiene que

$$
\left\|f_{t t}\right\|_{L^{2}} \leq C\left\|_{w}\right\|_{Y_{w}}^{2}+C\|w\|_{Y_{w}}^{2}
$$

Por lo tanto deducimos que $f \in Y_{f}$ y existe una constante $C>0$ dependiendo sólo de constantes de inmersión tal que

$$
\|f\|_{Y_{f}} \leq C\left\|_{w}\right\|_{Y_{w}}^{2}
$$

Con relación a $g$, usando el Teorema 4.1 y del hecho que $w \in H_{0}^{2}$ tenemos

$$
g=u_{x} w_{x}+\frac{1}{2}\left(w_{x}\right)^{3} \in H_{0}^{1}+H_{0}^{1} \text {, entonces } g \in C\left([0, T], H_{0}^{1}\right)
$$

Asimismo tenemos que

$$
\|g\|_{H^{1}} \leq C\|u\|_{H^{2}}\|w\|_{H^{2}}+C\|w\|_{H^{2}}^{3}
$$

luego como $u \in H^{2} \cap H_{0}^{1}, w \in H^{3} \cap H_{0}^{2}$ y $g \in H_{0}^{1}$ entonces existe una constante $C>0$ tal que

$$
\|g\|_{H_{0}^{1}} \leq C\left\|_{u}\right\|_{Y_{u}}\|w\|_{Y_{w}}+C\left\|_{w}\right\|_{Y_{w}}^{3}
$$

Derivando $g$ en relación a $t$, tenemos

$$
g_{t}=u_{x t} w_{x}+u_{x} w_{x t}+\frac{3}{2}\left(w_{x}\right)^{2} w_{x t}
$$

Usando el Teorema 4.1 y el Lema 4.1 tenemos que $u_{x t} w_{x} \in L^{2}, u_{x} w_{x t} \in H^{1},\left(w_{x}\right)^{2} w_{x t} \in H^{1}$, por lo tanto

y además

$$
g_{t} \in C\left([0, T], L^{2}\right)
$$

$$
\left\|g_{t}\right\|_{L^{2}} \leq C\left\|u_{t}\right\|_{H^{1}}\|w\|_{H^{2}}+C\|u\|_{H^{1}}\left\|w_{t}\right\|_{H^{2}}+\|w\|_{H^{2}}^{2}\left\|w_{t}\right\|_{H^{2}}
$$


Usando las características de $u$ y $w$ tenemos que existe una constante $C>0$ dependiendo de constantes de inmersión tal que

$$
\left\|g_{t}\right\|_{L^{2}} \leq C\|u\|_{Y_{u}}\|w\|_{Y_{w}}+C\left\|_{w}\right\|_{Y_{w}}^{3}
$$

Con lo que finalmente concluimos la demostración.

\section{Existencia de Solución local}

En esta sección demostraremos que el sistema $(\mathrm{P})$ posee una única solución en un intervalo de tiempo pequeño. Para obtener el resultado haremos uso del Teorema de Punto Fijo en conjunción con las propiedades de las soluciones de los problemas linealizados (P1) y (P2) y propiedades de las expresiones no lineales.

Dados $\left(u_{0}, u_{1}\right) \in H^{2} \cap H_{0}^{1} \times H_{0}^{1}$ y $\left(w_{0}, w_{1}\right) \in H^{3} \cap H_{0}^{2} \times H_{0}^{2}$ consideremos $Y=Y_{u} \times Y_{w}$ definimos el conjunto $K$ por

$$
K=\left\{\left(\begin{array}{c}
\tilde{u} \\
\tilde{w}
\end{array}\right) \in Y,\left(\begin{array}{c}
\tilde{u}(0) \\
\tilde{u}_{t}(0)
\end{array}\right)=\left(\begin{array}{c}
u_{0} \\
u_{1}
\end{array}\right),\left(\begin{array}{c}
\tilde{w}(0) \\
\tilde{w}_{t}(0)
\end{array}\right)=\left(\begin{array}{c}
w_{0} \\
w_{1}
\end{array}\right) \text {, talque }\left\|\left(\begin{array}{c}
\tilde{u} \\
\tilde{w}
\end{array}\right)\right\|_{Y} \leq R\right\}
$$

donde $R \leq 1$. De la estructura de $K$ vemos que este conjunto es una bola cerrada del espacio de Banach $Y_{u} \times Y_{w}$.

Definamos sobre $K$ una aplicación $\mathcal{P}$ tal que

$$
\begin{gathered}
\mathcal{P}: K \rightarrow Y_{u} \times Y_{w}, \text { tal que } \\
\left(\begin{array}{c}
\tilde{u} \\
\tilde{w}
\end{array}\right) \rightarrow\left(\begin{array}{c}
u \\
w
\end{array}\right),
\end{gathered}
$$

tal que a cada $\{\tilde{u}, \tilde{w}\}$ le hace corresponder el par $\{u, w\}$, donde $u$ y $w$ son soluciones los problemas linealizados (P1) y (P2) respectivamente, con $f$ y $g$ definidos como en (4.25) a partir de $\{\tilde{u}, \tilde{w}\}$. Luego por los Teoremas 3.1 y 3.2 este operador está bien definido. Además $\mathcal{P}$ es tal que $\mathcal{P}: K \rightarrow K$ y es una contracción. Lo que mostramos a continuación.

I) $\mathcal{P}: K \rightarrow K$. Sea $(\tilde{u}, \tilde{w}) \in Y_{u} \times Y_{w}$, luego si $u$ y $w$ son soluciones de los problemas linealizados $(\mathrm{P} 1)$ y $(\mathrm{P} 2)$ respectivamente, con $f$ y $g$ definidos como en (4.25) a partir de $\{\tilde{u}, \tilde{w}\}$, entonces de las estimativas obtenidas para las soluciones $u$ y $w$ en los Teoremas 3.1 y 3.2 , y haciendo uso del Teorema 4.2 deducimos que

$$
\begin{gathered}
\|u(t)\|_{H^{2} \cap H_{0}^{1}}+\left\|u_{t}(t)\right\|_{H_{0}^{1}}+\left\|u_{t t}(t)\right\|_{L^{2}} \leq\left\|u_{1}\right\|_{H_{0}^{1}}+\left\|u_{0}\right\|_{H^{2} \cap H_{0}^{1}}+\|f(0)\|_{H_{0}^{1}}+ \\
+C \int_{0}^{t}\|\tilde{w}\|_{Y_{w}}^{2} d s, \forall t \in[0, T],
\end{gathered}
$$


de donde se obtiene

$$
\|u\|_{Y_{u}} \leq\left\|u_{1}\right\|_{H_{0}^{1}}+\left\|u_{0}\right\|_{H^{2} \cap H_{0}^{1}}+\|f(0)\|_{H_{0}^{1}}+C T\|\tilde{w}\|_{Y_{w}}^{2} d s
$$

Asimismo para $w$ tenemos que

$$
\|w\|_{Y_{w}} \leq\left\|w_{1}\right\|_{H_{0}^{2}}+\left\|A w_{0}\right\|_{H_{0}^{1}}+\|g(0)\|_{L^{2}}+C T\left(\|\tilde{u}\|_{Y_{u}}\|\tilde{w}\|_{Y_{w}}+C\|\tilde{w}\|_{Y_{w}}^{3}\right) .
$$

Sumando estos dos resultados obtenemos

$$
C_{0}=\left\|u_{1}\right\|_{H_{0}^{1}}+\left\|u_{0}\right\|_{H^{2} \cap H_{0}^{1}}+\|f(0)\|_{H_{0}^{1}}+\left\|w_{1}\right\|_{H_{0}^{2}}+\left\|A w_{0}\right\|_{H_{0}^{1}}+\|g(0)\|_{L^{2}} .
$$

Podemos ahora definir $R \geq 1$ tal que $C_{0} \leq \frac{R}{2}$. Ahora como $\tilde{u}$ y $\tilde{w}$ son elementos de $Y_{u} \times Y_{w}$, entonces se sigue que

$$
\|u\|_{Y_{u}}+\|w\|_{Y_{w}} \leq \frac{R}{2}+C T\left(R^{2}+R^{2}+R^{3}\right) \leq \frac{R}{2}+3 C T R^{3}
$$

Si exigimos ahora que $T \leq \frac{1}{6 C R^{2}}$, entonces se tiene

$$
\left\|\mathcal{P}\left(\begin{array}{c}
\tilde{u} \\
\tilde{w}
\end{array}\right)\right\|_{Y_{u} \times Y_{w}}=\left\|\left(\begin{array}{c}
u \\
w
\end{array}\right)\right\|_{Y_{u} \times Y_{w}} \leq R .
$$

Lo que comprueba que para un intervalo de tiempo pequeño, $\mathcal{P}(K) \subset K$.

II) $\mathcal{P}$ es una contracción. Para demostrar esta propiedad de $\mathcal{P}$ tomamos un par de elementos de $K$. Sean

$$
\left(\begin{array}{c}
\tilde{u} \\
\tilde{w}
\end{array}\right),\left(\begin{array}{c}
\tilde{U} \\
\tilde{W}
\end{array}\right) \in K
$$

Luego mediante $\mathcal{P}$ corresponden a estos, respectivamente

$$
\left(\begin{array}{l}
u \\
w
\end{array}\right),\left(\begin{array}{c}
U \\
W
\end{array}\right) \in K
$$

tal que

$$
\mathcal{P}\left(\begin{array}{c}
\tilde{u} \\
\tilde{w}
\end{array}\right)=\left(\begin{array}{c}
u \\
w
\end{array}\right) \text { y } \mathcal{P}\left(\begin{array}{c}
\tilde{U} \\
\tilde{W}
\end{array}\right)=\left(\begin{array}{c}
U \\
W
\end{array}\right) .
$$

Definimos las funciones: 


$$
\begin{aligned}
& f=\frac{1}{2}\left(\tilde{w}_{x}\right)^{2}, g=\left(\tilde{u}_{x}+\frac{1}{2}\left(\tilde{w}_{x}\right)^{2}\right) \tilde{w}_{x}, \\
& F=\frac{1}{2}\left(\tilde{W}_{x}\right)^{2}, G=\left(\tilde{U}_{x}+\frac{1}{2}\left(\tilde{W}_{x}\right)^{2}\right) \tilde{W}_{x} .
\end{aligned}
$$

El objetivo es estimar las diferencias de $u$ con $U$ y de $w$ con $W$.

Para las ecuaciones en $u$ y $U$ tenemos:

Primera estimativa. Aplicando el Teorema 2.1 en particular la ecuación (2.3) para datos iniciales nulos, tenemos

$$
\left\|u_{t}(t)-U_{t}(t)\right\|_{H_{0}^{1}}+\left\|u_{t t}(t)-U_{t t}(t)\right\|_{L^{2}} \leq \int_{0}^{t}\left\|f_{x t}(s)-F_{x t}(s)\right\|_{L^{2}} d s .
$$

Estimemos $\left\|f_{t}(s)-F_{t}(s)\right\|_{H^{1}}$. Tenemos

$$
f_{t}-F_{t}=\frac{1}{2}\left(\tilde{w}_{t x}\right)^{2}-\frac{1}{2}\left(\tilde{W}_{t x}\right)^{2}=\frac{1}{2}\left(\tilde{w}_{t x}-\tilde{W}_{t x}\right)^{2}\left(\tilde{w}_{t x}+\tilde{W}_{t x}\right) .
$$

Entonces por el Teorema 4.1

$$
\left\|f_{t}(s)-F_{t}(s)\right\|_{H^{2}} \leq C\left\|\tilde{w}_{t}-\tilde{W}_{t}\right\|_{H^{2}}\left\|\tilde{w}_{t}+\tilde{W}_{t}\right\|_{H^{1}} \leq C\|\tilde{w}-\tilde{W}\|_{Y_{w}} R
$$

Luego tenemos

$$
\left\|u_{t}(t)-U_{t}(t)\right\|_{H_{0}^{1}}+\left\|u_{t t}(t)-U_{t t}(t)\right\|_{L^{2}} \leq T R C\|\tilde{w}-\tilde{W}\|_{y_{w}}
$$

Segunda estimativa. De las ecuaciones iniciales para $u$ y $\tilde{U}$, tenemos

$$
\begin{aligned}
& -u_{x x}=-u_{t t}+f_{x} \\
& -U_{x x}=-U_{t t}+F_{x} .
\end{aligned}
$$

usando (5.27) tenemos que

$$
\|u-U\|_{H^{2} \cap H_{0}^{1}} \leq T R C\|\tilde{w}-\tilde{W}\|_{Y_{w}}+\|f-F\|_{H^{1}} .
$$

Como $f(0)=F(0)$ tenemos que

$$
\|f-F\|_{H^{1}} \leq \int_{0}^{t}\left\|f_{t}(s)-F_{t}(s)\right\|_{H^{1}} d s
$$

Luego usando (5.26) tenemos que

$$
\|f(t)-F(t)\|_{H^{1}} \leq C T R\|\tilde{w}-\tilde{W}\|_{Y_{w}}
$$


Por lo tanto de (5.29) en (5.28)

$$
\|u(t)-U(t)\|_{H^{2} \cap H_{0}^{1}} \leq T R C\|\tilde{w}-\tilde{W}\|_{Y_{w}}
$$

Finalmente de (5.30) y (5.27) deducimos que

$$
\|u-U\|_{Y_{u}} \leq T R C\|\tilde{w}-\tilde{W}\|_{Y_{w}} .
$$

Para las ecuaciones en $w$ y $W$ tenemos

I Estimativa. Aplicando el Teorema 2.1 en particular la desigualdad (2.3) para datos iniciales nulos, y de las definiciones de $\tilde{g}$ y $\tilde{G}$ tenemos que

$$
\left\|w_{t}(t)-W_{t}(t)\right\|_{H_{0}^{2}}+\left\|w_{t t}(t)-W_{t t}(t)\right\|_{H_{0}^{1}} \leq C \int_{0}^{t}\left\|g_{t}(s)-G_{t}(s)\right\|_{L^{2}} d s
$$

Calculemos ahora la diferencia

$$
g_{t}-G_{t}=\left[\left(\tilde{u}_{x}+\frac{1}{2}\left(\tilde{w}_{x}\right)^{2}\right) \tilde{w}_{x}\right]_{t}-\left[\left(\tilde{U}_{x}+\frac{1}{2}\left(\tilde{W}_{x}\right)^{2}\right) \tilde{W}_{x}\right]_{t}
$$

luego

$$
g_{t}-G_{t}=\left(\tilde{u}_{x t} \tilde{w}_{x}-\tilde{U}_{x t} \tilde{W}_{x}\right)+\left(\tilde{u}_{x} \tilde{w}_{x t}-\tilde{U}_{x} \tilde{W}_{x t}\right)+\left(\frac{3}{2}\left(\tilde{w}_{x}\right)^{2} \tilde{w}_{x t}-\frac{3}{2}\left(\tilde{W}_{x}\right)^{2} \tilde{W}_{x t}\right)
$$

Sumando y restando términos adecuados en cada sumando de (5.33), obtenemos para el primer sumando

$$
\left\|\tilde{u}_{x t} \tilde{w}_{x}-U_{x t} \tilde{W}_{x}\right\|_{L^{2}} \leq C R\|\tilde{u}-\tilde{U}\|_{Y_{u}}+C R\|\tilde{w}-\tilde{W}\|_{Y_{w}}
$$

Similarmente para el otro sumando de (5.33) tenemos que

$$
\left\|\tilde{u}_{x} \tilde{w}_{x t}-\tilde{U}_{x} \tilde{W}_{x t}\right\|_{L^{2}} \leq C R\|\tilde{u}-\tilde{U}\|_{Y_{u}}+C R\|\tilde{w}-\tilde{W}\|_{Y_{w}}
$$

Para el tercer sumando en (5.33) tenemos

$$
\left\|\left(\tilde{w}_{x}\right)^{2} \tilde{w}_{x t}-\left(\tilde{W}_{x}\right)^{2} \tilde{W}_{x t}\right\|_{L^{2}} \leq C R^{2}\|\tilde{w}-\tilde{W}\|_{Y_{w}}
$$

Tomando la norma $L^{2}$ en (5.33) y usando las estimativas (5.34), (5.35) y (5.36) tenemos

$$
\left\|g_{t}(t)-G_{t}(t)\right\|_{L^{2}} \leq C R^{2}\|\tilde{w}-\tilde{W}\|_{Y_{w}}+C R\|\tilde{u}-\tilde{U}\|_{Y_{u}}
$$




$$
\left\|w_{t}(t)-W_{t}(t)\right\|_{H_{0}^{2}}+\left\|w_{t t}(t)-W_{t t}(t)\right\|_{H_{0}^{1}} \leq C T R^{2}\|\tilde{w}-\tilde{W}\|_{Y_{w}}+C R T\|\tilde{u}-\tilde{U}\|_{Y_{u}}
$$

II Estimativa. De las ecuaciones originales, en la forma variacional y de la definición de $\tilde{g}$ definido por $g$ y $\tilde{G}$ definido por $G$, luego para todo $\psi \in H_{0}^{2}$ tenemos

$$
\left(w_{x x}-W_{x x}, \psi_{x x}\right)=\left(-w_{t t}+W_{t t}+\tilde{g}-\tilde{G}, \psi\right)+\left(\left(-w_{t t}+W_{t t}+\tilde{g}-\tilde{G}\right)_{x}, \psi_{x}\right)
$$

Como $-w_{t t}+W_{t t}+\tilde{g}-\tilde{G} \in H_{0}^{1}$, por interpolación, se tiene que

$$
\|w-W\|_{H^{3} \cap H_{0}^{2}} \leq C\left\|w_{t t}-W_{t t}\right\|_{H_{0}^{1}}+C\|\tilde{g}-\tilde{G}\|_{H_{0}^{1}},
$$

Como en (3.21) mostramos que

$$
\|\tilde{g}-\tilde{G}\|_{H_{0}^{1}} \leq C\|g-G\|_{L^{2}},
$$

y del hecho que $g(0)=G(0)$ entonces

$$
\|w-W\|_{H^{3} \cap H_{0}^{2}} \leq C\left\|w_{t t}-W_{t t}\right\|_{H_{0}^{1}}+C \int_{0}^{t}\left\|g_{t}(s)-G_{t}(s)\right\|_{L^{2}} d s .
$$

Usando (5.37) y (5.38) en la desigualdad anterior tenemos

$$
\|w-W\|_{H^{3} \cap H_{0}^{2}} \leq C T R^{2}\|\tilde{w}-\tilde{W}\|_{Y_{w}}+C R T\|\tilde{u}-\tilde{U}\|_{Y_{u}} \cdot
$$

Finalmente de (5.38) y (5.39) tenemos

$$
\|w-W\|_{Y_{w}} \leq C T R^{2}\|\tilde{w}-\tilde{W}\|_{Y_{w}}+C R T\|\tilde{u}-\tilde{U}\|_{Y_{u}} .
$$

Por lo tanto de (5.31) y (5.40) tenemos

$$
\left\|\mathcal{P}\left(\begin{array}{c}
\tilde{u} \\
\tilde{w}
\end{array}\right)-\mathcal{P}\left(\begin{array}{c}
\tilde{U} \\
\tilde{W}
\end{array}\right)\right\|_{Y_{u} \times Y_{w}} \leq C T R^{2}\left\|\left(\begin{array}{c}
\tilde{u} \\
\tilde{w}
\end{array}\right)-\left(\begin{array}{c}
\tilde{U} \\
\tilde{W}
\end{array}\right)\right\|_{Y_{u} \times Y_{w}}
$$

Luego para un $T$ que $C T R^{2} \leq \frac{1}{2}$ mostramos que $\mathcal{P}$ es una contracción.

Por lo tanto, como $\mathcal{P}$ es una contracción en una bola cerrada $K$ de un espacio de Banach $Y_{u} \times Y_{w}$, existe un único punto

$$
\left(\begin{array}{l}
u \\
w
\end{array}\right) \in K, \text { tal que } \mathcal{P}\left(\begin{array}{l}
u \\
w
\end{array}\right)=\left(\begin{array}{l}
u \\
w
\end{array}\right),
$$

el que a su vez constituye solución local y única del problema $(\mathrm{P})$ en un intervalo de tiempo suficientemente pequeño. 


\section{Existencia de solución global}

En esta sección mostraremos que la solución local $\{u, w\}$ del problema $(\mathrm{P})$ puede extender su dominio de definición a todo el intervalo $[0, \infty)$. Utilizaremos para tal objetivo herramientas clásicas de continuidad de soluciones y estimativas a priori sobre la solución obtenida.

El proceso por el cual mostramos la existencia de una única solución local $\{u, w\}$ definida en un intervalo de tiempo suficientemente pequeño, digamos $\left[0, T_{1}\right]$, nos induce a iterar esta construcción. Tomando como datos iniciales las funciones $u\left(T_{1}\right), u_{t}\left(T_{1}\right)$ y $w\left(T_{1}\right), w_{t}\left(T_{1}\right)$, podemos mostrar que el problema $(\mathrm{P})$ admite una única solución definida en un intervalo de solución $[0, \delta]$. "Unimos" estas dos funciones, ver [3], y obtenemos una solución de (P) definida sobre $\left[0, T_{2}\right]$ con $T_{2}=T_{1}+\delta$. Definimos ahora la secuencia creciente $\left(T_{n}\right)_{n \geq 1}$; $\sin$ embargo puede suceder que $\sup _{n \geq 1} T_{n}<\infty$, ver [12].

Intervalo maximal de existencia. Sean $T_{1}<T_{2}$ y $\left\{u_{1}, w_{1}\right\}$ y $\left\{u_{2}, w_{2}\right\}$ dos soluciones fuertes de $(\mathrm{P})$ sobre $\left[0, T_{1}\right]$ y $\left[0, T_{2}\right]$ respectivamente. Por unicidad tenemos que $\left\{u_{1}, w_{1}\right\}=\left\{u_{2}, w_{2}\right\}$ sobre $\left[0, T_{1}\right]$. Consideramos ahora la familia $\left(u_{i}, w_{i}\right)_{i \in I}$ de todas las soluciones fuertes de (P) definida en algún intervalo $\left[0, T_{i}\right]$. Definimos

$$
T_{\max }=\sup _{i \in I} T_{i}
$$

Entonces $T_{m}$ puede ser finito $o+\infty$. Definimos las funciones $\{u, w\}$ sobre $\left[0, T_{\max }\right)$ por

$$
\{u, w\}=\left\{u_{i}, w_{i}\right\} \text {, si } t \in\left[0, T_{i}\right], i \in I \text {. }
$$

Esta función está bien definida por la unicidad en cada intervalo $\left[0, T_{i}\right]$. Esta solución es llamada solución maximal de (P).

Además de todos estos argumentos tenemos lo siguiente, ver [12], si $\{u, w\}$ es una solución maximal del problema $(\mathrm{P})$ entonces sólo una de las dos opciones siguientes es satisfecha:

A) $T_{\max }=+\infty$

$\left.A_{2}\right) T_{\max }<+\infty$ y en este caso

$\lim _{\mathrm{t} \rightarrow \mathrm{T}_{\max }}\left(\|u(t)\|_{H^{2} \cap H_{0}^{1}}+\left\|u_{t}(t)\right\|_{H_{0}^{1}}+\left\|u_{t t}(t)\right\|_{L^{2}}+\|w(t)\|_{H^{3} \cap H_{0}^{2}}+\left\|w_{t}(t)\right\|_{H_{0}^{2}}+\left\|w_{t t}(t)\right\|_{H_{0}^{1}}=\infty\right.$

Es obvio que si satisface $A_{1}$ entonces $\{u, w\}$ es una solución global. En el segundo caso $A_{2}$, decimos que solución $\{u, w\}$ explota en tiempo finito.

Para el segundo caso $A_{2}$, el límite necesariamente explota, pues si existe una sucesión $\left(t_{j}\right)_{j}$ tal que cuando $t_{j} \rightarrow T_{\max }$, entonces existe una constante $N>0$ tal que 


$$
\left\|u\left(t_{j}\right)\right\|_{H^{2} \cap H_{0}^{1}}+\left\|u_{t}\left(t_{j}\right)\right\|_{H_{0}^{1}}+\left\|u_{t t}\left(t_{j}\right)\right\|_{L^{2}}+\left\|w\left(t_{j}\right)\right\|_{H^{3} \cap H_{0}^{2}}+\left\|w_{t}\left(t_{j}\right)\right\|_{H_{0}^{2}}+\left\|w_{t t}\left(t_{j}\right)\right\|_{H_{0}^{1}} \leq N
$$

Entonces definimos el conjunto

$$
K=\left\{\left(\begin{array}{c}
\tilde{u} \\
\tilde{w}
\end{array}\right) \in Y,\left(\begin{array}{c}
\tilde{u}(0) \\
\tilde{u}_{t}(0)
\end{array}\right)=\left(\begin{array}{c}
u\left(t_{j}\right) \\
u_{t}\left(t_{j}\right)
\end{array}\right),\left(\begin{array}{c}
\tilde{w}(0) \\
\tilde{w}_{t}(0)
\end{array}\right)=\left(\begin{array}{c}
w\left(t_{j}\right) \\
w_{t}\left(t_{j}\right)
\end{array}\right),\left\|\left(\begin{array}{c}
\tilde{u} \\
\tilde{w}
\end{array}\right)\right\|_{Y} \leq R\right\}
$$

donde $R \geq 1, N$. Tomando $\left(u\left(t_{j}\right), u_{t}\left(t_{j}\right)\right)$ y $\left(w\left(t_{j}\right), w_{t}\left(t_{j}\right)\right)$ como datos iniciales, encontramos una única solución $\{\tilde{u}, \tilde{w}\}$ definida sobre un intervalo $[0, \delta]$, con $\delta$ dependiendo sólo de R. Juntando $\{u, w\}$ con $\{\tilde{u}, \tilde{w}\}$ obtenemos una solución local de $(\mathrm{P})$ definida sobre $\left[0, t_{j}+\delta\right]$. Para un $j$ suficientemente grande, $t_{j}+\delta>T_{\max }$, y esto es imposible, pues $\left[0, T_{\max }\right)$ es el intervalo maximal de solución.

De esta forma, para demostrar la existencia global de solución del problema $(\mathrm{P})$ es suficiente mostrar que $A_{2}$ no sucede, para lo cual obtendremos estimativas a priori de la solución $\{u, w\}$.

Teorema 6.1 Sea $T<T_{\max }$, considerando $\{u, w\}$ la solución maximal del sistema $(P)$ sobre el intervalo $[0, T]$, entonces existe una constante $M(T)>0$, de carácter exponencial, tal que $\forall t \in[0, T]$,

$$
\|u(t)\|_{H^{2} \cap H_{0}^{1}}+\left\|u_{t}(t)\right\|_{H_{0}^{1}}+\left\|u_{t t}(t)\right\|_{L^{2}}+\|w(t)\|_{H^{3} \cap H_{0}^{2}}+\left\|w_{t}(t)\right\|_{H_{0}^{2}}+\left\|w_{t t}(t)\right\|_{H_{0}^{1}} \leq M(T)
$$

La demostración del Teorema 6.1 sigue de los Lemas 6.1 y 6.2 que a continuación enunciamos y demostramos.

Lema 6.1 Con las hipótesis del Teorema 6.1, existe una constante $M(T)>0$, tal que

$$
\left\|u_{t}(t)\right\|_{H_{0}^{1}}+\left\|u_{t t}(t)\right\|_{L^{2}}+\left\|w_{t}(t)\right\|_{H_{0}^{2}}+\left\|w_{t t}(t)\right\|_{H_{0}^{1}} \leq M(T), \forall t \in[0, T]
$$

Demostración. De la definición de solución fuerte, tenemos

$$
\left\{\begin{array}{c}
\left(u_{t t}, \phi\right)+\left(\left[u_{x}+\frac{1}{2}\left(w_{x}\right)^{2}\right], \phi_{x}\right)=0, \forall \phi \in H_{0}^{1} \\
\left(w_{t t}, \psi\right)+\left(w_{x t t}, \psi_{x}\right)+\left(w_{x x}, \psi_{x x}\right)+\left(w_{x}\left(u_{x}+\frac{1}{2}\left(w_{x}\right)^{2}\right), \psi_{x}\right)=0, \forall \psi \in H_{0}^{2} .
\end{array}\right.
$$

Derivando las ecuaciones con respecto a $t$ y después tomando $\phi=u_{t t}$ y $\psi=w_{t t}$, tenemos

$$
\begin{aligned}
& \frac{1}{2} \frac{d}{d t}\left\|u_{t t}\right\|^{2}+\left(u_{x t}+w_{x} w_{x t}, u_{x t t}\right)=0, \\
& \frac{1}{2} \frac{d}{d t}\left\|w_{t t}\right\|^{2}+\frac{1}{2} \frac{d}{d t}\left\|w_{x t t}\right\|^{2}+\frac{1}{2} \frac{d}{d t}\left\|w_{t x x}\right\|^{2}+
\end{aligned}
$$




$$
+\left(w_{x t}\left(u_{x}+\frac{1}{2}\left(w_{x}\right)^{2}\right), w_{t t x}\right)+\left(w_{x}\left(u_{t x}+w_{x} w_{x t}\right), w_{x t t}\right)=0
$$

Observación. Una demostración más rigurosa de (6.41) y (6.42) puede seguir el siguiente camino,

- Considerar las ecuaciones del sistema (P) en los tiempos $t+h$ y $t-h$ respectivamente y tomar la diferencia.

- Dividir por $2 \mathrm{~h}$, tomar el producto interno con $u_{t t} \mathrm{y} w_{t t}$ respectivamente y tomar el límite cuando h converge a cero.

Sumando ambas ecuaciones (6.41) y (6.42), y observando que

$$
\begin{gathered}
\left(w_{x}\left(u_{t x}+w_{x} w_{x t}\right), w_{x t t}\right)=\left(u_{t x}+w_{x} w_{x t}, w_{x} w_{x t t}\right) \\
\left(w_{x t}\left(u_{x}+\frac{1}{2}\left(w_{x}\right)^{2}\right), w_{t t x}\right)=\left(u_{x}+\frac{1}{2}\left(w_{x}\right)^{2}, w_{t t x} w_{x t}\right)
\end{gathered}
$$

entonces tenemos

$$
\begin{gathered}
\frac{1}{2} \frac{d}{d t}\left\|u_{t t}\right\|+\frac{1}{2} \frac{d}{d t}\left\{\left\|w_{t t}\right\|^{2}+\left\|w_{t t x}\right\|^{2}+\left\|w_{t x x}\right\|^{2}\right\}+ \\
+\left(u_{x t}+w_{x} w_{x t}, u_{x t t}+w_{x} w_{x t t}\right)+\left(u_{x}+\frac{1}{2}\left(w_{x}\right)^{2}, w_{t t x} w_{x t}\right)=0 .
\end{gathered}
$$

Estudiemos la expresión

$$
X=\left(u_{x t}+w_{x} w_{x t}, u_{x t t}+w_{x} w_{x t t}\right)+\left(w_{x t}\left(u_{x}+\frac{1}{2}\left(w_{x}\right)^{2}\right), w_{t t x}\right)
$$

Sumando y sustrayendo $w_{x t} w_{x t}$, dentro del primer producto interno de $X$, tenemos

$$
X=\left(\frac{d}{d t}\left(u_{x t}+w_{x} w_{x t}\right), u_{x t}+w_{x} w_{x t}\right)-\left(u_{x t}+w_{x} w_{x t}, w_{x t} w_{x t}\right)+\left(u_{x}+\frac{1}{2}\left(w_{x}\right)^{2}, w_{t t x} w_{x t}\right)
$$

Ahora consideremos la expresión

$$
Z=-\left(u_{x t}+w_{x} w_{x t}, w_{x t} w_{x t}\right)+\left(u_{x}+\frac{1}{2}\left(w_{x}\right)^{2}, w_{t t x} w_{x t}\right)
$$

Sumando y sustrayendo a $Z$ la expresión $\left(u_{x t}+w_{x} w_{x t}, \frac{1}{2}\left(w_{x t}\right)^{2}\right)$, entonces

$$
Z=\frac{d}{d t}\left(u_{x}+\frac{1}{2}\left(w_{x}\right)^{2}, \frac{1}{2}\left(w_{x t}\right)^{2}\right)-\frac{3}{2}\left(u_{x t}+w_{x} w_{x t},\left(w_{x t}\right)^{2}\right) .
$$

Colocando este resultado en $X$, tenemos que

$$
X=\left(\frac{d}{d t}\left(u_{x t}+w_{x} w_{x t}\right), u_{x t}+w_{x} w_{x t}\right)+\frac{d}{d t}\left(u_{x}+\frac{1}{2}\left(w_{x}\right)^{2}, \frac{1}{2}\left(w_{x t}\right)^{2}\right)-\frac{3}{2}\left(u_{x t}+w_{x} w_{x t},\left(w_{x t}\right)^{2}\right)
$$


Observemos ahora que

$$
X=\frac{1}{2} \frac{d}{d t}\left\|u_{x t}+w_{x} w_{x t}\right\|^{2}+\frac{1}{2} \frac{d}{d t}\left(u_{x}+\frac{1}{2}\left(w_{x}\right)^{2},\left(w_{x t}\right)^{2}\right)-\frac{3}{2}\left(u_{x t}+w_{x} w_{x t},\left(w_{x t}\right)^{2}\right)
$$

Por lo tanto, volviendo a (6.43), con este resultado para $X$, tenemos

$$
\begin{gathered}
\frac{1}{2} \frac{d}{d t}\left\|u_{t t}\right\|^{2}+\frac{1}{2} \frac{d}{d t}\left\{\left\|w_{t t}\right\|^{2}+\left\|w_{t t x}\right\|^{2}+\left\|w_{t x x}\right\|^{2}\right\}+ \\
+\frac{1}{2} \frac{d}{d t}\left\|u_{x t}+w_{x} w_{x t}\right\|^{2}+\frac{1}{2} \frac{d}{d t}\left(u_{x}+\frac{1}{2}\left(w_{x}\right)^{2},\left(w_{x t}\right)^{2}\right)-\frac{3}{2}\left(u_{x t}+w_{x} w_{x t},\left(w_{x t}\right)^{2}\right)=0 .
\end{gathered}
$$

Integrando de 0 a $t \leq T$,

$$
\begin{gathered}
\left.\frac{1}{2}\left\{\left\|u_{t t}\right\|^{2}+\left\|w_{t t}\right\|^{2}+\left\|w_{t t x}\right\|^{2}+\left\|w_{t x x}\right\|^{2}\right\}\right|_{0} ^{t}+ \\
+\left.\frac{1}{2}\left\|u_{x t}+w_{x} w_{x t}\right\|^{2}\right|_{0} ^{2}+\left.\frac{1}{2}\left(u_{x}+\frac{1}{2}\left(w_{x}\right)^{2},\left(w_{x t}\right)^{2}\right)\right|_{0} ^{t}= \\
=\frac{3}{2} \int_{0}^{t}\left(u_{x t}+w_{x} w_{x t},\left(w_{x t}\right)^{2}\right) d s .
\end{gathered}
$$

Definamos:

$$
\begin{aligned}
& Q=\frac{1}{2}\left\{\left\|u_{t t}\right\|^{2}+\left\|w_{t t}\right\|^{2}+\left\|w_{t t x}\right\|^{2}+\left\|w_{t x x}\right\|^{2}\right\}+ \\
& +\frac{1}{2}\left\|u_{x t}+w_{x} w_{x t}\right\|^{2}+\frac{1}{2}\left(u_{x}+\frac{1}{2}\left(w_{x}\right)^{2},\left(w_{x t}\right)^{2}\right) .
\end{aligned}
$$

Entonces,

$$
\begin{aligned}
\mid Q-\frac{1}{2}\left\{\left\|u_{t t}\right\|^{2}\right. & \left.+\left\|w_{t t}\right\|^{2}+\left\|w_{t t x}\right\|^{2}+\left\|w_{t x x}\right\|^{2}\right\}+\frac{1}{2}\left\|u_{x t}+w_{x} w_{x t}\right\|^{2} \mid= \\
& =\left|\frac{1}{2}\left(u_{x}+\frac{1}{2}\left(w_{x}\right)^{2},\left(w_{x t}\right)^{2}\right)\right| .
\end{aligned}
$$

Sobre el último término, para $\varepsilon>0$,

$$
\mid\left(u_{x}+\frac{1}{2}\left(w_{x}\right)^{2},\left(w_{x t}\right)^{2} \mid \leq\left\|u_{x}+\frac{1}{2}\left(w_{x}\right)^{2}\right\|\left\|\left(w_{x t}\right)^{2}\right\| \leq \frac{1}{2 \varepsilon}+\left\|u_{x}+\frac{1}{2}\left(w_{x}\right)^{2}\right\|^{2}+\frac{\varepsilon}{2}\left\|\left(w_{x t}\right)^{2}\right\|^{2}\right.
$$

Como la energía es constante y usando los resultados de productos de funciones de espacios de Sobolev enunciados en la sección 4 , tenemos

$$
\mid\left(u_{x}+\frac{1}{2}\left(w_{x}\right)^{2},\left(w_{x t}\right)^{2} \mid \leq \frac{1}{2 \varepsilon} E(0)+\frac{\varepsilon}{2}\left\|w_{x t}\right\|_{L^{2}}\left\|w_{x t}\right\|_{H_{0}^{1}} \leq \frac{1}{2 \varepsilon} E(0)+\frac{\varepsilon}{2} E(0)\left\|w_{t}\right\|_{H_{0}^{2}}\right.
$$

Por lo tanto, 
75

$$
\begin{gathered}
\left|Q-\frac{1}{2}\left\{\left\|u_{t t}\right\|^{2}+\left\|w_{t t}\right\|^{2}+\left\|w_{t t x}\right\|^{2}+\left\|w_{t x x}\right\|^{2}\right\}-\frac{1}{2}\left\|u_{x t}+w_{x} w_{x t}\right\|^{2}\right| \leq \\
\leq \frac{1}{2 \varepsilon} E(0)+\frac{\varepsilon}{2} E(0)\left\|w_{t}\right\|_{H_{0}^{2}} .
\end{gathered}
$$

luego

$$
\begin{gathered}
-\frac{1}{2 \varepsilon} E(0)-\frac{\varepsilon}{2} E(0)\left\|w_{t}\right\|_{H_{0}^{2}} \\
+\frac{1}{2}\left\{\left\|u_{t t}\right\|^{2}+\left\|w_{t t}\right\|^{2}+\left\|w_{t t x}\right\|^{2}+\left\|w_{t x x}\right\|^{2}\right\}+\frac{1}{2}\left\|u_{x t}+w_{x} w_{x t}\right\|^{2} \leq Q \leq \\
\leq \frac{1}{2}\left\{\left\|u_{t t}\right\|^{2}+\left\|w_{t t}\right\|^{2}+\left\|w_{t t x}\right\|^{2}+\left\|w_{t x x}\right\|^{2}\right\}+\frac{1}{2}\left\|u_{x t}+w_{x} w_{x t}\right\|^{2}+ \\
+\frac{1}{2 \varepsilon} E(0)+\frac{\varepsilon}{2} E(0)\left\|w_{t}\right\|_{H_{0}^{2}} .
\end{gathered}
$$

y para un $\varepsilon$ suficientemente pequeño, entonces existe una constante $C>0$ tal que

$$
-C E(0)+C\left\{\left\|u_{t t}\right\|^{2}+\left\|w_{t t}\right\|^{2}+\left\|w_{t t x}\right\|^{2}+\left\|w_{t x x}\right\|^{2}\right\}+\frac{1}{2}\left\|u_{x t}+w_{x} w_{x t}\right\|^{2} \leq Q
$$

Volviendo a (6.44) y por la definición de $Q$, tenemos

$$
Q(t)=\frac{3}{2} \int_{0}^{t}\left(u_{x t}+w_{x} w_{x t},\left(w_{x t}\right)^{2}\right) d s+Q(0)
$$

y usando (6.45), se tiene que

$$
\begin{gathered}
C\left\{\left\|u_{t t}\right\|^{2}+\left\|w_{t t}\right\|^{2}+\left\|w_{t t x}\right\|^{2}+\left\|w_{t x x}\right\|^{2}\right\}+\frac{1}{2}\left\|u_{x t}+w_{x} w_{x t}\right\|^{2} \leq \\
\leq \frac{3}{2} \int_{0}^{t}\left(u_{x t}+w_{x} w_{x t},\left(w_{x t}\right)^{2}\right) d s+Q(0)+C E(0) .
\end{gathered}
$$

Observemos,

$$
\left|\left(u_{x t}+w_{x} w_{x t},\left(w_{x t}\right)^{2}\right)\right| \leq\left\|u_{x t}+w_{x} w_{x t}\right\|\left\|\left(w_{x t}\right)^{2}\right\| \leq \frac{1}{2}\left\|u_{x t}+w_{x} w_{x t}\right\|^{2}+\frac{1}{2}\left\|\left(w_{x t}\right)^{2}\right\|^{2}
$$

Entonces, por los resultados de productos de funciones de espacios de Sobolev, y como la energía es constante, tenemos

$$
\left|\left(u_{x t}+w_{x} w_{x t},\left(w_{x t}\right)^{2}\right)\right| \leq \frac{1}{2}\left\|u_{x t}+w_{x} w_{x t}\right\|_{L^{2}}^{2}+C E(0)\left\|w_{t}\right\|_{H_{0}^{2}}^{2} .
$$

Por lo tanto de (6.47) en (6.46), tenemos

$$
\begin{aligned}
& C\left\{\left\|u_{t t}\right\|^{2}+\left\|w_{t t}\right\|^{2}+\left\|w_{t t x}\right\|^{2}+\left\|w_{t x x}\right\|^{2}\right\}+\frac{1}{2}\left\|u_{x t}+w_{x} w_{x t}\right\|^{2} \leq \\
& C \int_{0}^{t}\left(\frac{1}{2}\left\|u_{x t}+w_{x} w_{x t}\right\|_{L^{2}}^{2}+C E(0)\left\|w_{t}\right\|_{H_{0}^{2}}^{2}\right) d s+Q(0)+C E(0) .
\end{aligned}
$$


Sea $C_{0}=Q(0)+C E(0)$. Entonces existe una constante $C>0$ tal que

$$
\begin{aligned}
& \left\|u_{t t}\right\|^{2}+\left\|w_{t t}\right\|_{H_{0}^{1}}^{2}+\left\|w_{t}\right\|_{H_{0}^{2}}^{2}+\left\|u_{x t}+w_{x} w_{x t}\right\|^{2} \leq \\
& \leq C \int_{0}^{t}\left\|u_{x t}+w_{x} w_{x t}\right\|_{L^{2}}^{2} d s+C \int_{0}^{t}\left\|w_{t}\right\|_{H_{0}^{2}}^{2} d s+C C_{0}
\end{aligned}
$$

Luego, por la desigualdad de Gronwall,

$$
\left\|u_{t t}\right\|^{2}+\left\|w_{t t}\right\|_{H_{0}^{1}}^{2}+\left\|w_{t}\right\|_{H_{0}^{2}}^{2}+\left\|u_{x t}+w_{x} w_{x t}\right\|^{2} \leq C C_{0} \exp (T)
$$

Por otro lado,

$$
\left(u_{x t}\right)^{2} \leq 2\left(u_{x t}+w_{x} w_{x t}\right)^{2}+2\left(w_{x} w_{x t}\right)^{2}
$$

Entonces,

$$
\int_{0}^{L}\left(u_{x t}\right)^{2} d x \leq 2 \int_{0}^{L}\left(u_{x t}+w_{x} w_{x t}\right)^{2} d x+2 \int_{0}^{L}\left(w_{x} w_{x t}\right)^{2} d x
$$

Luego, de (6.48) y de los resultados de productos de funciones de espacios de Sobolev,

$$
\int_{0}^{L}\left(u_{x t}\right)^{2} d x \leq C \exp (T)+C\left\|w_{x}\right\|_{H_{0}^{1}}^{2}\left\|w_{x t}\right\|_{L^{2}}^{2} \leq C \exp (T)+C\left\|_{w}\right\|_{H_{0}^{2}}^{2}\left\|w_{t}\right\|_{H_{0}^{1}}^{2} \text {. }
$$

Entonces como la energía es constante, tenemos

$$
\int_{0}^{L}\left(u_{x t}\right)^{2} d x \leq C \exp (T)+C(E(0))^{2}
$$

luego de este último resultado y de (6.48), tenemos la existencia de una constante $M(T)>0$ de carácter exponencial, tal que

$$
\left\|u_{t t}\right\|_{L^{2}}^{2}+\left\|u_{t}\right\|_{H_{0}^{1}}^{2}+\left\|w_{t t}\right\|_{H_{0}^{1}}^{2}+\left\|w_{t}\right\|_{H_{0}^{2}}^{2} \leq M(T)
$$

Con lo que queda demostrado el Lema.

Lema 6.2 Si se verifican las hipótesis del Teorema 6.1, entonces existe una constante $M(T)>0$, tal que

$$
\|u(t)\|_{H^{2} \cap H_{0}^{1}}+\|w(t)\|_{H^{3} \cap H_{0}^{2}} \leq M(T), \forall t \in[0, T] .
$$

Demostración. De la ecuación inicial, para las oscilaciones longitudinales, tenemos

$$
-u_{x x}=-u_{t t}-\frac{1}{2}\left[\left(w_{x}\right)^{2}\right]_{x} \in L^{2}
$$

tomando la norma de $L^{2}$ a ambos lados, entonces por el Lema $6.1 \mathrm{y}$ de los resultados de productos de funciones de espacios de Sobolev 


$$
\left\|u_{x x}\right\|_{L^{2}}=\left\|u_{t t}\right\|_{L^{2}}+C\left\|w_{x} w_{x x}\right\|_{L^{2}} \leq M(T)+C\left\|w_{x}\right\|_{H_{0}^{1}}\left\|w_{x x}\right\|_{L^{2}} \leq M(T)+C\left\|_{w}\right\|_{H_{0}^{2}}^{2} .
$$

De donde deducimos que

$$
\|u\|_{H^{2} \cap H_{0}^{1}}^{2} \leq M(T)+C E(0) .
$$

Por otro lado, para la variable $w$, de la ecuación inicial, tenemos

$$
\left(w_{x x}, \psi_{x x}\right)=\left(-w_{t t}, \psi\right)+\left(-w_{x t t}, \psi_{x}\right)+\left(-w_{x}\left(u_{x}+\frac{1}{2}\left(w_{x}\right)^{2} \psi_{x}\right)\right) \forall \psi \in H_{0}^{2} .
$$

Sea, $g=w_{x}\left(u_{x}+\frac{1}{2}\left(w_{x}\right)^{2}\right)$, luego como mostrado anteriormente, existe $\tilde{g}$ tal que

$$
\left(w_{x x}, \psi_{x x}\right)=\left(-w_{t t}, \psi\right)+\left(-w_{x t t}, \psi_{x}\right)+(-\tilde{g}, \psi)+\left(-\tilde{g}_{x}, \psi_{x}\right), \forall \psi \in H_{0}^{2} .
$$

Luego

$$
\left(w_{x x}, \psi_{x x}\right)=\left(-w_{t t},-\tilde{g}, \psi\right)+\left(-w_{x t t},-\tilde{g}_{x}, \psi_{x}\right) \forall \psi \in H_{0}^{2} .
$$

Como $-w_{t t}-\tilde{g} \in H_{0}^{1}$, entonces por interpolación

$$
\|w\|_{H^{3} \cap H_{0}^{2}} \leq\left\|-w_{t t}-\tilde{g}\right\|_{H_{0}^{1}} .
$$

y de la definición de $\tilde{g}$, tenemos

$$
\|w\|_{H^{3} \cap H_{0}^{2}} \leq\left\|w_{t t}\right\|_{H_{0}^{1}}+\|g\|_{L^{2}},
$$

es decir

$$
\|w\|_{H^{3} \cap H_{0}^{2}} \leq\left\|w_{t t}\right\|_{H_{0}^{1}}+\| w_{x}\left(u_{x}+\frac{1}{2}\left(w_{x}\right)^{2} \|_{L^{2}} .\right.
$$

Usando los resultados de productos de funciones de espacios de Sobolev tenemos

$$
\| w_{x}\left(u_{x}+\frac{1}{2}\left(w_{x}\right)^{2}\left\|_{L^{2}} \leq C\right\| w_{x}\left\|_{H_{0}^{1}}\right\| u_{x}+\frac{1}{2}\left(w_{x}\right)^{2} \|_{L^{2}} \leq C E(0)^{\frac{1}{2}} E(0)^{\frac{1}{2}} .\right.
$$

Por lo tanto se tiene que

$$
\|w\|_{H^{3} \cap H_{0}^{2}} \leq M(T)+C E(0)
$$

Luego de (49) y (50) se obtiene el resultado enunciado.

\section{Unicidad de solución}

En esta sección demostraremos que la solución global del problema (P) es única. Para este objetivo haremos uso de estimativas directamente sobre las soluciones, es decir usaremos el método de la energía. 
Sean $\{U, W\}$ y $\{V, Z\}$ dos soluciones globales y fuertes del sistema (P) para los mismos datos iniciales, es decir, dado $T>0$ definiendo como $u=U-V$ y $w=W-Z$, tenemos que satisfacen para todo $\varphi \in H_{0}^{1}$ y para todo $\psi \in H_{0}^{2}$

$$
\left\{\begin{array}{l}
\left(u_{t t}, \varphi\right)+\left(u_{x}, \varphi_{x}\right)+\frac{1}{2}\left(\left(W_{x}\right)^{2}-\left(Z_{x}\right)^{2}, \varphi_{x}\right)=0, \\
\left(w_{t t}, \psi\right)+\left(w_{x t t}, \psi_{x}\right)+\left(w_{x x}, \psi_{x x}\right)+\left(W_{x}\left(U_{x}+\frac{1}{2}\left(W_{x}\right)^{2}\right)-Z_{x}\left(V_{x}+\frac{1}{2}\left(Z_{x}\right)^{2}\right), \psi_{x}\right)=0,
\end{array}\right.
$$

Sea $\varphi=u_{t}$ e $\psi=w_{t}$ e integrando ambas ecuaciones de 0 a $t \leq T$, tenemos

$$
\left\{\begin{array}{c}
\left\|u_{t}\right\|^{2}+\left\|u_{x}\right\|^{2}=-2 \int_{0}^{t}\left(\left(W_{x}\right)^{2}-\left(Z_{x}\right)^{2}, u_{t x}\right) d s \\
\left\|w_{t}\right\|^{2}+\left\|w_{x t}\right\|^{2}+\left\|w_{x x}\right\|^{2}=-2 \int_{0}^{t}\left(W_{x}\left(U_{x}+\frac{1}{2}\left(W_{x}\right)^{2}+Z_{x}\left(V_{x}+\frac{1}{2}\left(Z_{x}\right)^{2}\right), w_{x t}\right) d s\right.
\end{array}\right.
$$

Analicemos las siguientes expresiones en (51).

Sea

$$
X_{1}=-\int_{0}^{t}\left(\left(W_{x}\right)^{2}-\left(Z_{x}\right)^{2}, u_{t x}\right) d s
$$

Entonces de la integración por partes, tenemos

$$
X_{1}=-\left.\left(\left(W_{x}\right)^{2}-\left(Z_{x}\right)^{2}, u_{x}\right)\right|_{0} ^{t}+\int_{0}^{t}\left(\frac{d}{d t}\left(W_{x}\right)^{2}-\left(Z_{x}\right)^{2}, u_{x}\right) d s
$$

Como $u(0)=0$, entonces

$$
X_{1}=-\left(\left(W_{x}\right)^{2}-\left(Z_{x}\right)^{2}, u_{x}\right)+\int_{0}^{t}\left(\frac{d}{d t}\left(W_{x}\right)^{2}-\left(Z_{x}\right)^{2}, u_{x}\right) d s
$$

De la desigualdad de Cauchy - Schwarz y para $\varepsilon>0$,

$$
X_{1} \leq \frac{1}{2 \varepsilon}\left\|\left(W_{x}\right)^{2}-\left(Z_{x}\right)^{2}\right\|^{2}+\frac{\varepsilon}{2}\left\|u_{x}\right\|^{2}+\frac{1}{2} \int_{0}^{t}\left\|\frac{d}{d t}\left(W_{x}\right)^{2}-\left(Z_{x}\right)^{2}\right\| d s+\frac{1}{2} \int_{0}^{t}\left\|u_{x}\right\|^{2} d s
$$

De la expresión anterior, por los resultados de productos de espacios de Sobolev, tenemos

$$
\begin{gathered}
\left\|\left(W_{x}\right)^{2}-\left(Z_{x}\right)^{2}\right\| \leq C\left\|W_{x}-Z_{x}\right\|_{L^{2}}\left\|W_{x}+Z_{x}\right\|_{H^{1}} \leq \\
\leq C\left\|w_{x}\right\|_{L^{2}}\left(\|W\|_{H_{0}^{2}}+\|Z\|_{H_{0}^{2}}\right)=C\left(\|W\|_{H_{0}^{2}}+\|Z\|_{H_{0}^{2}}\right)\left\|\int_{0}^{t} w_{t x} d s\right\|_{L^{2}} \leq \\
\leq C\left(\|W\|_{H_{0}^{2}}+\|Z\|_{H_{0}^{2}}\right) \int_{0}^{t}\left\|w_{t x}\right\|_{L^{2}} d s \leq C\left(\|W\|_{H_{0}^{2}}+\|Z\|_{H_{0}^{2}}\right) T^{\frac{1}{2}}\left(\int_{0}^{t}\left\|w_{t x}\right\|_{L^{2}}^{2} d s\right)^{\frac{1}{2}} .
\end{gathered}
$$


Por lo tanto tenemos

$$
\frac{1}{2 \varepsilon}\left\|\left(W_{x}\right)^{2}-\left(Z_{x}\right)^{2}\right\|_{L^{2}}^{2} \leq C\left(\|W\|_{H_{0}^{2}}+\|Z\|_{H_{0}^{2}}\right)^{2} T \int_{0}^{t}\left\|w_{t x}\right\|_{L^{2}}^{2} d s .
$$

Luego por el Teorema 6.1 existen constantes $M_{1}(T)>0$ y $M_{2}(T)>0$ dependiendo de $T$ tal que Por lo tanto tenemos

$$
\frac{1}{2 \varepsilon}\left\|\left(W_{x}\right)^{2}-\left(Z_{x}\right)^{2}\right\|_{L^{2}}^{2} \leq C\left(M_{1}(T)+M_{2}(T)\right)^{2} T \int_{0}^{t}\left\|w_{t x}\right\|_{L^{2}}^{2} d s
$$

Por otro lado, de (52), la expresión

$$
\frac{d}{d t}\left(\left(W_{x}\right)^{2}-\left(Z_{x}\right)^{2}\right)=2\left(W_{x} W_{t x}-Z_{x} Z_{t x}\right)=2\left(W_{x}-Z_{x}\right) W_{t x}+2 Z_{x}\left(W_{t x}-Z_{t x}\right)
$$

Entonces por los resultados de productos de funciones de espacios de Sobolev, tenemos

$$
\left\|\frac{d}{d t}\left(W_{x}\right)^{2}-\left(Z_{x}\right)^{2}\right\|_{L^{2}} \leq C\left\|w_{x}\right\|_{H_{0}^{1}}\left\|W_{t x}\right\|_{L^{2}}+C\left\|Z_{x}\right\|_{H_{0}^{1}}\left\|w_{x t}\right\|_{L^{2}}
$$

Luego por el Teorema 6.1 existen las constantes $M_{1}(T)>0$ y $M_{2}(T)>0$, dependiendo de $T$, tal que

$$
\int_{0}^{t}\left\|\frac{d}{d t}\left(W_{x}\right)^{2}-\left(Z_{x}\right)^{2}\right\|_{L^{2}}^{2} d s \leq M_{1}^{2}(T) \int_{0}^{t}\left\|w_{x}\right\|_{H^{1}}^{2} d s+M_{2}^{2}(T) \int_{0}^{t}\left\|w_{x t}\right\|_{L^{2}}^{2} d s
$$

Por lo tanto de (53) y (54) en (52)

$$
\begin{gathered}
X_{1} \leq C\left(M_{1}(T)+M_{2}(T)\right)^{2} T \int_{0}^{t}\left\|w_{t x}\right\|_{L^{2}}^{2} d s+ \\
+\frac{\varepsilon}{2}\left\|u_{x}\right\|^{2}+M_{1}^{2}(T) \int_{0}^{t}\left\|w_{x}\right\|_{H_{0}^{1}}^{2} d s+M_{2}^{2}(T) \int_{0}^{t}\left\|w_{x t}\right\|_{L^{2}}^{2} d s+\frac{1}{2} \int_{0}^{t}\left\|u_{x}\right\|^{2} d s .
\end{gathered}
$$

Luego, de (55) en (51) y para un $\varepsilon$ suficientemente pequeño, tenemos la existencia de una constante $C>0$ tal que

$$
\begin{gathered}
\left\|u_{t}\right\|^{2}+\|u\|_{H_{0}^{1}}^{2} \leq C\left(M_{1}(T)+M_{2}(T)\right)^{2} T \int_{0}^{t}\left\|w_{t}\right\|_{H_{0}^{2}}^{2} d s+ \\
+C M_{1}^{2}(T) \int_{0}^{t}\|w\|_{H_{0}^{2}}^{2} d s+C M_{2}^{2}(T) \int_{0}^{t}\left\|w_{t}\right\|_{H_{0}^{2}}^{2} d s+\frac{1}{2} \int_{0}^{t}\|u\|_{H_{0}^{1}}^{2} d s .
\end{gathered}
$$

De la segunda ecuación en (51),

$$
\begin{aligned}
& -\left(W_{x}\left(U_{x}+\frac{1}{2}\left(W_{x}\right)^{2}\right)-Z_{x}\left(V_{x}+\frac{1}{2}\left(Z_{x}\right)^{2}\right), w_{x t}\right) \leq \\
& \leq\left\|W_{x}\left(U_{x}+\frac{1}{2}\left(W_{x}\right)^{2}\right)-Z_{x}\left(V_{x}+\frac{1}{2}\left(Z_{x}\right)^{2}\right)\right\|\left\|w_{x t}\right\| .
\end{aligned}
$$


Sea

$$
\begin{gathered}
X_{2}=W_{x}\left(U_{x}+\frac{1}{2}\left(W_{x}\right)^{2}\right)-Z_{x}\left(V_{x}+\frac{1}{2}\left(Z_{x}\right)^{2}\right)=\left(W_{x} U_{x}-Z_{x} V_{x}\right)+\frac{1}{2}\left(\left(W_{x}\right)^{3}-\left(Z_{x}\right)^{3}\right) \\
=\left(U_{x}-V_{x}\right) W_{x}+\left(W_{x}-Z_{x}\right) V_{x}+\frac{1}{2}\left(W_{x}-Z_{x}\right)\left(\left(W_{x}\right)^{2}+W_{x} Z_{x}+\left(Z_{x}\right)^{2}\right) \\
=u_{x} W_{x}+w_{x} V_{x}+\frac{1}{2} w_{x}\left(\left(W_{x}\right)^{2}+W_{x} Z_{x}+\left(Z_{x}\right)^{2}\right)
\end{gathered}
$$

Entonces, aplicando los resultados de productos de funciones de espacios de Sobolev, tenemos

$$
\begin{gathered}
\left\|X_{2}\right\| \leq\left\|u_{x}\right\|_{L^{2}}\left\|W_{x}\right\|_{H^{1}}+\left\|w_{x}\right\|_{H^{1}}\left\|V_{x}\right\|_{L^{2}}+ \\
+\left\|w_{x}\right\|_{L^{2}}\left(\left\|\left(W_{x}\right)^{2}\right\|_{H^{1}}+\left\|W_{x} Z_{x}\right\|_{H^{1}}+\left\|\left(Z_{x}\right)^{2}\right\|_{H^{1}}\right)
\end{gathered}
$$

Luego, por el Teorema 6.1 existen $M_{1}(T)>0$ y $M_{2}(T)>0$, constantes dependiendo de $T$, tal que

$$
\left\|X_{2}\right\|_{L^{2}} \leq\left\|u_{x}\right\|_{L^{2}} M_{1}(T)+\left\|w_{x}\right\|_{H^{1}} M_{2}(T)+C\left\|w_{x}\right\|_{L^{2}} M_{1}(T) M_{2}(T) .
$$

Luego de este resultado en (57), existe entonces una constante $C>0$ tal que

$$
\begin{gathered}
-\left(W_{x}\left(U_{x}+\frac{1}{2}\left(W_{x}\right)^{2}-Z_{x}\left(U_{x}+\frac{1}{2}\left(Z_{x}\right)^{2}\right), w_{x t}\right) \leq\right. \\
\leq C M_{1}^{2}(T)\|u\|_{H_{0}^{1}}^{2}+C M_{2}^{2}(T)\|w\|_{H_{0}^{2}}^{2}+C M_{1}^{2}(T) M_{2}^{2}(T)\|w\|_{H_{0}^{2}}^{2}+C\left\|w_{t}\right\|_{H_{0}^{1}}^{2} .
\end{gathered}
$$

$\operatorname{De}(58)$ en (51),

$$
\begin{gathered}
\left\|w_{t}\right\|^{2}+\left\|w_{x t}\right\|^{2}+\left\|w_{x x}\right\|^{2} \leq C M_{1}^{2}(T) \int_{0}^{t}\|u\|_{H_{0}^{1}}^{2} d s+ \\
C M_{2}^{2}(T) \int_{0}^{t}\|w\|_{H_{0}^{2}}^{2} d s+C M_{1}^{2}(T) M_{2}^{2}(T) \int_{0}^{t}\|w\|_{H_{0}^{2}}^{2} d s+C \int_{0}^{t}\left\|w_{t}\right\|_{H_{0}^{1}}^{2} d s .
\end{gathered}
$$

Sumando los resultados (56) y (59), y considerando $C(T)$ una constante genérica, tenemos

$$
\begin{aligned}
\left\|u_{t}\right\|^{2}+ & \|u\|_{H_{0}^{1}}^{2}+\left\|w_{t}\right\|_{H_{0}^{1}}^{1}+\|w\|_{H_{0}^{2}}^{2} \leq C(T) \int_{0}^{t}\left\|w_{t}\right\|_{H_{0}^{1}}^{2} d s+ \\
& +C(T) \int_{0}^{t}\|w\|_{H_{0}^{2}}^{2} d s+C(T) \int_{0}^{t}\|u\|_{H_{0}^{1}}^{2} d s .
\end{aligned}
$$

Por lo tanto existe una constante $C(T)>0$, tal que

$$
\left\|u_{t}\right\|^{2}+\|u\|_{H_{0}^{1}}^{2}+\left\|w_{t}\right\|_{H_{0}^{1}}^{2}+\|w\|_{H_{0}^{2}}^{2} \leq M(T) \int_{0}^{t}\left\{\left\|u_{t}\right\|^{2}+\|u\|_{H_{0}^{1}}^{2}+\left\|w_{t}\right\|_{H_{0}^{1}}^{2}+\|w\|_{H_{0}^{2}}^{2}\right\} d s
$$


De la desigualdad de Gronwall se sigue que $u \equiv 0, w \equiv 0$. Por lo tanto la solución global del sistema (P) es única.

Observación: Usando el mismo método por el cual mostramos la unicidad del sistema (P), podemos demostrar que la solución depende continuamente de los datos iniciales. Ante esta propiedad, podemos decir que el sistema (P) es un problema bien puesto en el sentido de Hadamard para soluciones fuertes.

\section{Observaciones finales}

Una pregunta inmediata sería sobre la existencia de soluciones débiles para el modelo estudiado, éstas se obtendrán por argumentos de densidad.

Podemos también considerar una distribución de temperatura desconocida sobre la viga, acoplado al sistema (1), actuando directamente sobre los desplazamientos verticales, usando las mismas técnicas usadas aquí, es posible mostrar la existencia de soluciones para el modelo termoelástico. Asimismo podemos mostrar que este modelo se aproxima de forma débil a un modelo de viga termoelástico de Timoshenko. Sin embargo está en abierto encontrar una condición necesaria y suficiente para que esta convergencia sea fuerte en la norma de la energía.

Considerando términos lineales de disipación actuando en todo el dominio (viga) en el modelo (1), obtenemos un sistema no lineal de viga disipativo, haciendo uso del mismo método abordado aquí, mostramos que el problema tiene solución única, con técnicas de perturbación de la energía podemos mostrar la estabilización exponencial de la energía y con los resultados de aproximación débil obtenemos la estabilización de un modelo disipativo de viga de Timoshenko.

\section{REFERENCIAS BIBLIOGRÁFICAS}

[1] ADAMS, R.A. Sobolev spaces. Academic Press. New York (1975).

[2] BREZIS, H. Análisis funcional. Teoría y aplicaciones. Alianza Editorial, Madrid, (1984).

[3] CAZENAVE, T. and HARAUX, A. Introduction aux problèmes d'évolution semi-linèaires. Mathematics \& Applications. Èditions ELLIPSES, Paris (1990).

[4] GILBART, D. and TRUDINGER, N. S. Elliptic partial differential equations of second order. A series of comprehensive studies in mathematics. Springer Verlag. Berlin, (1983).

[5] HARAUX, A. Semi-Linear hyperbolic problems in bounded domains. Mathematical Reports, Hardwood, London (1987).

[6] VON KÁRMAN, T. Festigkeitprobleme in Maschinenbau Encyklopedie der Mathemastichen Wissenschaften 4, pp. 314-385, (1910).

[7] KESAVAN, S. Topics in functional analysis and applications. John Wiley \& sons, (1989).

[8] KOCH, H. and LASIECKA, I. Hadamard wellposedness of weak solutions in nonlinear dynamic elasticity - full von Kárman systems. Progress in Nonlinear Differential Equations and their Applications. 50, pp. 197-216, (2002).

[9] LAGNESE, J. E. and LEUGERING., G. Uniform stabilization of a nonlinear beam by nonlinear boundary feedback. Journal of Differential Equations. 91, 355 - 388 (1991). 
[10] LASIECKA, I. Weak, classical, and intermediate solutions to full von Kárman system of dynamic nonlinear elasticity. Applicable Analysis, 68, 121 - 145 (1998).

[11] MAGENES, J. L. and MAGENES, E. Problèms aux limites non homogènes et applications. Vol. I. DUNOD, Paris (1968).

[12] PAZY, A. Semigroups of linear operators and applications to partial differential equations. Applied Mathematics Sciences, Springer Verlag, New York, (1983).

[13] PERLA MENZALA, G. and ZUAZUA, E. The beam equation as a limit of a 1-D nonlinear von Kárman model. Applied Math. Letters 12.47 - 52, (1999).

[14] PERLA MENZALA, G. and ZUAZUA, E. Timoshenko's beam equation as limit of a nonlinear one-dimensional von Kárman system. Proc. Royal Soc. Edimburg. 130 A, (2000).

[15] PERLA MENZALA, G. and ZUAZUA, E. Timoshenko's plate equations as a singular limit of the dinamical von Kárman system. J. Math. Pures et Appli. 79 pp. 73 - 94, (2000).

[16] PUEL, J. P. and TUCSNAK, M. Global existence for the full von Kárman system. Appl. Math. Optim. 34: 139 - 160 (1996). 